BULLETIN (New Series) OF THE

AMERICAN MATHEMATICAL SOCIETY

Volume 47, Number 3, July 2010, Pages 373-417

S 0273-0979(10)01295-4

Article electronically published on March 10, 2010

\title{
TODAY'S MENU: GEOMETRY AND RESOLUTION OF SINGULAR ALGEBRAIC SURFACES
}

\author{
E. FABER AND H. HAUSER
}

\begin{abstract}
The courses are Triviality, Tangency, Transversality, Symmetry, Simplicity, Singularity. These characteristic local plates serve as our invitation to algebraic surfaces and their resolution. Please take a seat.
\end{abstract}

\section{Contents}

\begin{tabular}{l|l|} 
Appetizers & 374 \\
Ingredients & 376 \\
Triviality (soup) & 377 \\
Tangency (salad) & 379 \\
Transversality (fish) & 380 \\
Symmetry (roast) & 380 \\
Simplicity (mousse au chocolat) & 381 \\
Singularity (digestif) & 386 \\
Triviality: Whitney umbrella $x^{2}=y^{2} z$ & 390 \\
Tangency: Kolibri $y^{2}=x^{2} z^{2}+x^{3}$ & 393 \\
Transversality: Iris, $x^{2} y+y^{2} z=z^{4}$ & 399 \\
Symmetry: Helix $x^{4}+y^{2} z^{2}=x^{2}$ & 406 \\
Simplicity: Sofa $x^{2}+y^{3}+z^{5}=0$ & 412 \\
Singularity: Daisy $\left(x^{2}-y^{3}\right)^{2}=\left(z^{2}-y^{2}\right)^{3}$ & 413 \\
Appendix: Basic concepts & 413 \\
Stratification & 414 \\
Blowup & 415 \\
Resolution & 415 \\
Acknowledgments & 415 \\
About the authors & References
\end{tabular}

Received by the editors July 1, 2009, and, in revised form, October 20, 2009.

2000 Mathematics Subject Classification. Primary 14E15; Secondary 32S45.

Both authors have been supported by the Austrian Science Fund (FWF) in the frame of the projects P18992 and P21461. E. F. has been supported by grant F-443 of the University of Vienna. This paper is written for people not necessarily familiar with the advanced techniques of algebraic geometry. Experts are invited to browse through the article for many pictures and a few scattered open problems.

(C)2010 American Mathematical Society Reverts to public domain 28 years from publication 
Our menu consists of six geometric phenomena related to the resolution of singularities of algebraic surfaces. The courses are Triviality (soup), Tangency (salad), Transversality (fish), Symmetry (roast), Simplicity (dessert) and Singularity (digestif). In each course a selected singular surface will illustrate these concepts. On the way, we will resolve the surface and depict its resolution process graphically. After some appetizers we present the basic ingredients of our dinner. For the cooking we will mostly use algebraic food. To keep the appetite alive, the more technical definitions (of singularities, blowups, resolution, normal crossings, ...) are relegated to the appendix (after the meal). Nonetheless we provide a quick guide to the most important notions (without proof or further explanation) that will be used in the text. The pictures appearing later in the article will give vivid illustrations of these notions.

\section{Appetizers}
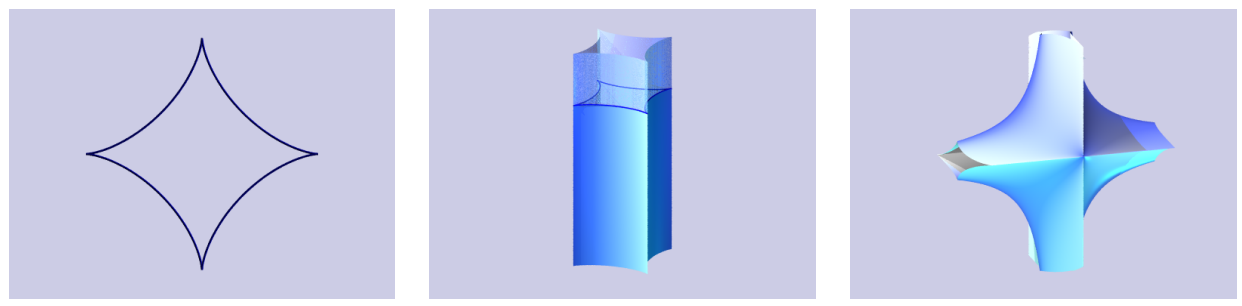

Figure 1. Genesis of Astrix.

The Astroid is the real plane curve $C$ in $\mathbb{R}^{2}$ that is traced by a marked point on a circle rolling inside a circle of four times its radius. The trajectory of the point has the parametrization $t \rightarrow\left(\cos ^{3} t, \sin ^{3} t\right)$. Alternatively, it can be given by the implicit equation with rational exponents $f: x^{2 / 3}+y^{2 / 3}=1$. Raising powers and manipulating we obtain from $f$ the polynomial equation $27 x^{2} y^{2}=$ $\left(1-x^{2}-y^{2}\right)^{3}$. The Astroid is a closed Hypocycloid with four cusp-like singular points; the symmetries are those of a square, say the dihedral group $D_{2}$ (Figure 1 . left).

Take the Cartesian product of the Astroid with the $z$-axis in $\mathbb{R}^{3}$. The resulting surface is a cylinder in $\mathbb{R}^{3}$ with the same equation as the Astroid, but now considered as an equation in three variables (Figure 1, middle). In this equation replace the variable $y$ by the product $y z$. The result is $27 x^{2} y^{2} z^{2}=\left(1-x^{2}-y^{2} z^{2}\right)^{3}$, which defines the surface Astrix in $\mathbb{R}^{3}$ (Figure 1, right).

Later on we will ask how to resolve surfaces $X$ such as Astrix. By this we mean to find a smooth surface $X^{\prime}$ together with a projection onto $X$ that is an isomorphism outside the singular locus of $X$. It is thus a parametrization of the singular surface by a manifold. We may ask additionally that the symmetries of $X$ lift to $X^{\prime}$, i.e., that the projection is equivariant. This is already less evident.

The Node in $\mathbb{R}^{2}$ is defined by the cubic equation $y^{2}=x^{3}+x^{2}$. It looks like the Greek character $\alpha$. Take again the cylinder over this curve in $\mathbb{R}^{3}$. Along the vertical $z$-axis it is singular; its local geometry there consists of two planes intersecting transversally (see Figure 2, left).

Modify this construction by varying the size of the horizontal Node as it rises along the $z$-axis. More specifically, the diameter of the loop shall equal the square 
of the height $z$. The respective equation is $y^{2}=x^{2} z^{2}+x^{3}$ and defines a surface called Kolibri. At the origin it has a gusset-like shape (see Figure 2, right). The intersection with the $x y$-plane $z=0$ is the Cusp of equation $y^{2}=x^{3}$.
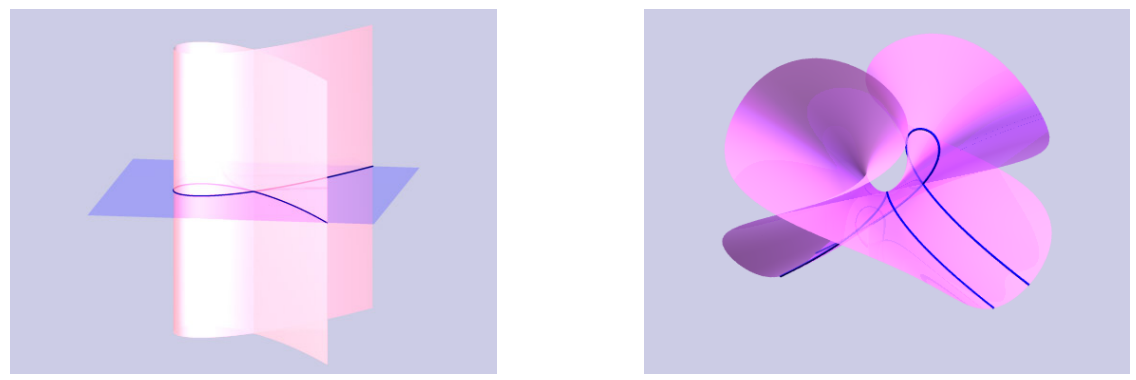

Figure 2. Construction of Kolibri.

A bug walking along the $z$-axis will observe that in a small neighborhood the singular shape of Kolibri develops smoothly as the angle between the two "planes" varies continuously. Arriving at the origin, the local geometry changes drastically. There, the singularity is much more involved. The origin is the most singular point, whereas Kolibri is equisingular along the $z$-axis if we stay off 0 . Later on this is made precise by means of a Whitney stratification of a variety. An equisingular stratification is a decomposition of the variety into smooth, locally closed subsets, called strata, such that the variety has the same type (in a concrete sense) along each stratum. One method to do this is to consider tangent planes at smooth points together with their limits as the points approach a singularity. The resulting stratification is very geometric and has an analytic counterpart, which is studied in the section Triviality.

The Cylinder over the circle is the zeroset of $x^{2}+y^{2}=1$, taken as an equation in three variables on $\mathbb{R}^{3}$; see Figure 4 , left. Substitute $x$ and $y$ by the fractions $(x-y z) /(y z)$ and $y^{2} / z$. After clearing denominators we get the equation $x^{2}+y^{6}=$ $2 x y z$, which defines a surface called Eighty (see Figure 3).

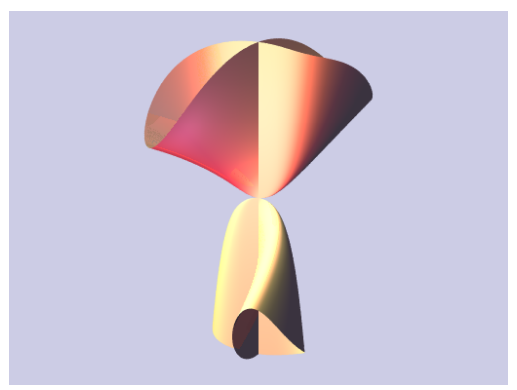

FiguRE 3. Eighty.

Eighty is smooth everywhere except along the $z$-axis. We should see it as the result of squeezing and deforming the Cylinder in a specific way. The algebra behind this geometric operation is the substitution from above. Later on we will 
reverse this operation by reconstructing the Cylinder from Eighty via blowups. By this we mean modifications on a variety that loosen its singularities and give the variety more space to unfold. Each blowup improves the singularities so that a finite number of them allow us to resolve the variety, i.e., transform it into a manifold. For Eighty, three blowups are needed, and each of them is of a very simple nature, e.g. given by a map such as $(x, y, z) \mapsto(x y, y, y z)$. The intermediate stages can be seen in Figure 4 ,
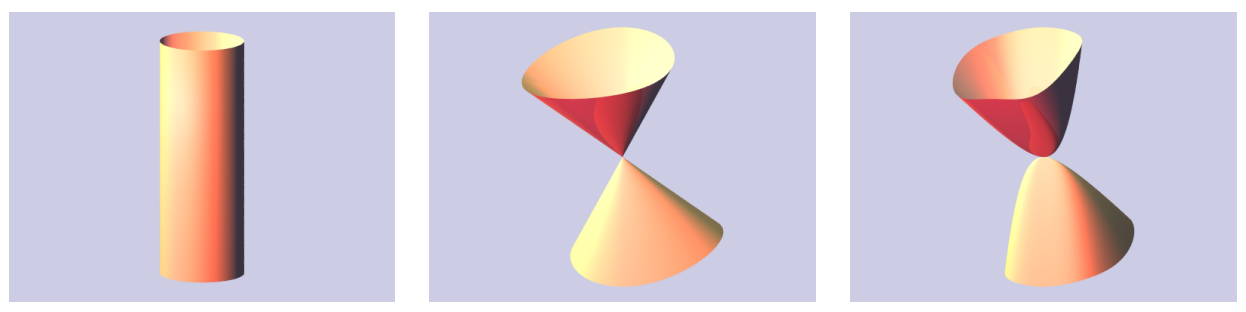

Figure 4. Construction of Eighty.

The composition of the three blowups turns out to be the map from $\mathbb{R}^{3}$ to $\mathbb{R}^{3}$ sending $(x, y, z)$ to $\left((x+1) y z^{2}, y z, y z^{2}\right)$. Indeed, one checks that it maps the Cylinder onto Eighty. Moreover, it is an isomorphism over the regular points of Eighty, hence a resolution. It will be our task to realize this procedure in all generality (for surfaces).

\section{INGREDIENTS}

The surfaces live in affine three-space $\mathbb{A}_{K}^{3}=K^{3}$, where $K$ is a field (of characteristic 0). Mostly we work over the ground field $\mathbb{R}$ of real numbers. Sometimes computations are carried out over $\mathbb{C}$. If so, it will be explicitly stated. Each of our surfaces is an algebraic variety, i.e., it is given as the zeroset of one polynomial in three variables. The points where a surface $X$ is locally a manifold are called smooth points. The remaining points of $X$ are the singular points of $X$; their collection is denoted by $\operatorname{Sing}(X)$. The singular locus of $X$ is always a closed proper subvariety of $X$. It therefore consists of curves and/or points or it is empty if $X$ is smooth. The interesting thing is to understand how $X$ comes together at its singular points. This is highly nontrivial and represents a major flavor of our meal.

The main idea to handle the singularities of a singular surface $X$ is to parametrize $X$ by a smooth surface. One tries to find a surjective $\operatorname{map} \varphi$ from a two-dimensional manifold $\widetilde{X}$ to $X$ such that $\varphi$ is almost everywhere an isomorphism. Then one can think of $X$ as the projection or contraction of the smooth surface $\widetilde{X}$ living in a higher-dimensional manifold down to $\mathbb{A}_{K}^{3}$. We call $\varphi: \widetilde{X} \rightarrow X$ a resolution of the singularities of $X$.

As we have already seen in the appetizers, the resolution map $\varphi$ can be written as a composition of simple maps, blowups. Additionally it satisfies some properties as explained in the appendix. The existence of a resolution $\varphi$ of a variety of arbitrary dimension over a field of characteristic zero was proven by Hironaka [18. For positive characteristic there is still no proof of the existence of a resolution in dimension $\geq 4$. 
Since we consider all surfaces embedded in $\mathbb{A}_{K}^{3}$, the resolution $\varphi: \widetilde{X} \rightarrow X$ should be induced by a morphism $\psi: \widetilde{\mathbb{A}}^{3} \rightarrow \mathbb{A}^{3}$ of some three-dimensional manifold $\widetilde{\mathbb{A}}^{3}$ onto $\mathbb{A}^{3}$. The surface $\widetilde{X}$ lives in $\widetilde{\mathbb{A}}^{3}$.

We now describe blowups, which make up the resolution map: these are certain birational proper morphisms and will be our most important tool to resolve a surface. A birational morphism is a map that is almost everywhere (on a Zariskidense subset) an isomorphism and proper means that the inverse image of a compact set is compact. A blowup is then a proper birational morphism $\pi: \widetilde{X} \rightarrow X$ which is associated in a specific way (see the appendix) to the choice of its center $Z$. The center, which is a closed subvariety of $X$, is the locus of points above which $\pi$ fails to be an isomorphism. The variety $\widetilde{X}$ is called the blowup of $X$. It turns out that the blowup map $\pi$ is induced by a blowup map $\tau: \widetilde{\mathbb{A}}^{3} \rightarrow \mathbb{A}^{3}$ of the ambient space with the same center: if the center $Z$ of a blowup of $\mathbb{A}^{3}$ is contained in $X$, then one can show that $\pi: \widetilde{X} \rightarrow X$, the blowup of $X$ along $Z$, is equal to $\left.\tau\right|_{\widetilde{X}}$, the restriction of the ambient blowup to $\tilde{X}$.

The exceptional locus or exceptional divisor of the blowup $\pi: \widetilde{X} \rightarrow X$ is the locus in $\widetilde{X}$ where the blowup is not an isomorphism. If we consider $Z \subseteq X$ embedded in $\mathbb{A}^{3}$ it is given as the inverse image $\mathbb{D}=\tau^{-1}(Z) \cap \widetilde{X}$ of the center, and the exceptional divisor of the ambient blowup is denoted by $\mathbb{E}=\tau^{-1}(Z)$. The total transform $X^{*}$ of $X$ under $\tau$ is the inverse image $\tau^{-1}(X)$. The strict transform $X^{\prime}$ of $X$ is the (Zariski) closure in $\widetilde{\mathbb{A}}^{3}$ of the total transform minus the exceptional locus, i.e., $X^{\prime}=\overline{\tau^{-1}(X \backslash Z)}$. The irreducible components of the intersection of the strict transform $X^{\prime}$ with the exceptional divisor $\mathbb{E}$, or equivalently, the components of $\mathbb{D}$, are called exceptional curves of the blowup of $X$.

The blowup $\widetilde{\mathbb{A}}^{3}$ can be covered by affine charts, i.e., by charts that are isomorphic to an (open subset of an) affine algebraic variety. From the blowup map $\tau: \widetilde{\mathbb{A}}^{3} \rightarrow \mathbb{A}^{3}$ one obtains chart expressions of $\tau$ that make it possible to write equations for the strict transform $X^{\prime}$ in each affine chart.

We start by providing a short description of the various courses.

Triviality (soup). Take a surface, pick a point on it and look at the geometry at this point, locally in a small neighborhood. For most choices of this point the geometry will be the same: flatland. These are the smooth points of the surface. Now take a singular point. If it is isolated there will be only finitely many similar points, and the local geometry at these points can be rather involved. If the singular locus of your surface contains a curve, pick a point on this curve. What is the local geometry of the surface at the chosen point? Two cases may occur: (a) The point is a singular point of the curve. Then it will have only finitely many "twin-points". (b) The point is a smooth point of the curve. Then it makes sense to observe the change of the local geometry of the surface as we move the point along the curve; see Figure 5. We study in this way the local singularity type of the surface along (a component of) its singular locus.

In the simplest case the surface is analytically isomorphic (locally at a fixed point $p$ of the singular curve $Y$ ) to the Cartesian product of the curve with a transversal cross section, i.e., the sections of the surface with a plane perpendicular to the curve. In this case one says that the surface $X$ is locally (analytically) trivial along $Y$ at $p$. 


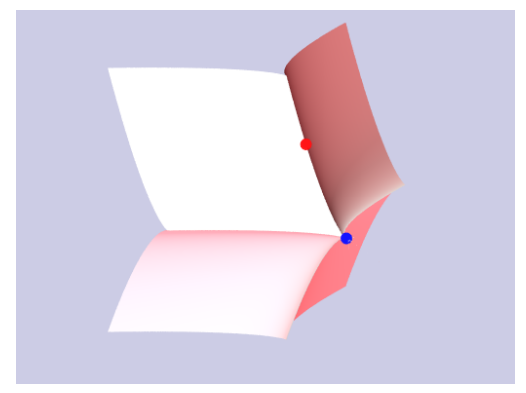

Figure 5. Points in the singular locus: type (a) blue and type (b) red.

For example, consider $X=V(x y z)$, the union of the three coordinate hyperplanes in $\mathbb{A}^{3}$. The singular locus of $X$ consists of the three coordinate axes. At each point on one of these axes, except the origin, $X$ is locally analytically isomorphic to the union of two planes that intersect transversally. The origin is a somewhat more singular point because here all three planes meet; $X$ looks different there.

We will explain how to decompose any surface $X$ into subsets along which it is locally trivial. Fix a point $p \in X$. To study the local nature of $X$ at $p$ one considers arbitrarily small open (Euclidean) neighbourhoods of $p$. This corresponds to taking the germ of $X$ at $p$. A theorem of Ephraim 8 states that, in any dimension, the locus $Y$ of points $q \in X$ such that the germs of $X$ in these points $q$ are analytically isomorphic to the germ of $X$ in $p$ is a submanifold of $X$. For surfaces, $Y$ can be the entire variety $X$ (if it is smooth), or a collection of curves and/or points. Furthermore, Ephraim proved that $X$ is locally isomorphic around $p$ to $Y \times Z$ for some germ $Z$. So we obtain a decomposition of the surface $X$ into its smooth points and (not necessarily finitely many) locally closed curves and points along which $X$ is analytically trivial.

The situation is much more interesting when $X$ is not locally analytically trivial along an arbitrarily given curve $Y$ on $X$. Still, the geometry of $X$ may be similar along $Y$. We then call $X$ equisingular along $Y$. There are several ways to interpret this phrase. One of the most used equisingularity criteria was given by Whitney [41] and is discussed below. Similarly one can find algebraic or topological equisingularity criteria; see Zariski [45] or Teissier [37, respectively.

Decomposing $X$ into locally closed submanifolds along which it is trivial or equisingular is an example of a rather general concept. A stratification of $X$ is a filtration $X=Z_{d} \supseteq Z_{d-1} \supseteq \cdots \supseteq Z_{0}$, where each $Z_{i}$ is an algebraic subset of $X$, i.e., Zariski-closed. The irreducible components $X_{\alpha}$ of $Z_{i}-Z_{i-1}$ are called the strata of $X$. The $X_{i}$ are differences of algebraic subsets and smooth, and we have $X=\bigcup_{\alpha} X_{\alpha}$. For a detailed description of a stratification, see the appendix.

Stratifications reflect the structure of a singular variety and can also be helpful for the resolution of singularities. Here the idea is to stratify the singular variety such that the strata measure the "intricacy" of the singularities. The stratum with the worst singularities can be chosen as the center for a blowup, which should then improve the singularities. However, we will see in the following that finding such a stratification is a nontrivial problem. 
Tangency (salad). In the second course we will again work with stratifications. We shall consider a very geometric stratification proposed by Whitney [4]. Suppose we have found a stratification $X=\bigcup_{\alpha \in A} X_{\alpha}$ of a surface. We call a pair of strata $\left(X_{\alpha}, X_{\beta}\right)$ with $X_{\beta} \subseteq \overline{X_{\alpha}} \backslash X_{\alpha}$ adjacent. Then it is interesting to observe how adjacent strata actually fit together.

Consider a stratum $X_{\alpha}$ on our surface $X$. How does this stratum "run into" an adjacent stratum $X_{\beta}$ ? Whitney gave two conditions that a stratification should satisfy: in condition (a) one considers limits of the tangent spaces of $X_{\alpha}$ along a sequence of points $\left\{x_{i}\right\}$ in $X_{\alpha}$ that approach a point $y \in X_{\beta}$. For condition (b) one also looks at the limit of the secant lines of two sequences $\left\{x_{i}\right\}$ in $X_{\alpha}$ and $\left\{y_{i}\right\} \in X_{\beta}$ that approach the point $y \in X_{\beta}$. Both conditions express the behaviour of $X_{\alpha}$ along $X_{\beta}$, locally at $y$, and are known as Whitney's conditions (a) and (b). If all pairs of adjacent strata satisfy Whitney's conditions, then one speaks of a Whitney stratification of $X$. A Whitney stratification always exists for any analytic variety (in $\mathbb{C}^{n}$ or $\mathbb{R}^{n}$ ); see [41. Such a stratification is a useful tool in studying equisingularity problems; see [38]. It is basic for many other constructions, for example intersection homology; see [11].

In the Tangency section we will give a more detailed description of Whitney's conditions and consider an example of a surface, Kolibri, where the first condition is satisfied whereas the second fails.

Transversality (fish). In this course we use the resolution of a flowery surface called Iris to discuss questions about transversality. In differential geometry, transversality between two smooth subvarieties of a manifold $M$ means that their tangent spaces at each point of the intersection span the whole tangent space of $M$ at that point. This notion clearly depends on the embedding in $M$. In our algebraic setting, transversality has to be seen in a more general perspective, with several options.

Two smooth algebraic varieties intersect cleanly if at each point of the intersection the intersection of the tangent spaces coincides with the tangent space of the intersection. For a subvariety of $\mathbb{A}^{n}$ with several components, the classical notion of transversality is that of normal crossings: We say a subvariety in $\mathbb{A}^{n}$ has normal crossings at a point $p$ if the variety is at $p$ locally isomorphic to the union of coordinates subspaces of $\mathbb{A}^{n}$. A variety has normal crossings if it has normal crossings at any of its points. If the variety consists of just two smooth components, a normal crossing is the same as the clean intersection of the two components. Two normal crossing subvarieties of $\mathbb{A}^{n}$ are said to meet transversally if their irreducible components have normal crossings at each point of their intersection.

These notions become relevant in the embedded resolution of singularities, where it is required that the total transform has normal crossings. This is equivalent to saying that the strict transform is smooth, that the exceptional divisor has normal crossings, and that both meet transversally in the above sense [7, 15. An embedded resolution is needed in Hironaka's proof of resolution [18, which uses induction over the embedding dimension of the variety that has to be resolved. It is easy to see that having normal crossings is preserved under blowups in smooth centers provided the variety and the center meet transversally. Amongst others, the embedded resolution is also important for the problem of finding compactifications of complex manifolds; see for example [10]. 
A subvariety of $\mathbb{A}^{n}$ is called mikado if it is locally at each of its points locally analytically isomorphic to an arbitrary union of linear spaces. This is clearly a more general concept than just having normal crossings. It is easier to achieve in the resolution process of a variety. However we will give an example showing that this property is not stable under blowups.

Symmetry (roast). In the next course we will discuss the concept of symmetry for our surfaces. Consider the group $G$ of automorphisms of $\mathbb{A}^{3}$ that fix a surface $X$ that is embedded in $\mathbb{A}^{3}$. The group $G$ is called the symmetry group of $X$. Here, we only take into account symmetries of finite order. In the case of $\mathbb{A}_{\mathbb{R}}^{3}$, the classification of finite linear symmetry groups is well known; see for example [25]. Except for the cyclic groups $C_{k} \cong \mathbb{Z}_{k}$, where $k \geq 1$ and $k \in \mathbb{N}$ and the dihedral groups $D_{k}$ with $k \geq 4$ one finds three exceptional groups $T, O$ and $I$, corresponding to the symmetry groups of the Platonic solids: Tetrahedron, Octahedron and Icosahedron, respectively.

In the appetizers, the surface Astrix, which is invariant under the action of $\mathbb{Z}_{2} \times D_{4}$, was constructed. Its defining polynomial $\left(x^{2}+y^{2} z^{2}-1\right)^{3}-27 x^{2} y^{2} z^{2}=0$ is invariant under the permutation of $y$ and $z$. The symmetrization of a polynomial obtained by inserting invariant polynomials instead of the variables may produce new singularities that come from the fixed points of the symmetry automorphism. It is somewhat mysterious how the symmetrization of a surface is related to its blowups.

The singular locus of a symmetric surface $X$ inherits the symmetries of $X$. This affects the choice of the center of blowups for the resolution: we require that the resolution preserves the symmetries of $X$. Such a resolution is then called equivariant. Usually, centers are smooth subvarieties of $X$ that lie in the singular locus of $X$. The singular locus of a symmetric surface $X$ consists in general of several components, namely curves and points that are permuted by the action of the symmetry group of $X$. We can simply choose one of these components as the center and start with blowing them up. This, however, may destroy the symmetries of $X$.

One way to preserve symmetries under blowups is to choose the intersection point of the singular curves as the center. It can be checked that the singularities of $X$ will not necessarily improve under this blowup. One can also try to blow up the whole singular locus of $X$ in one step. Then the symmetries of $X$ will be preserved. The only problem in this approach is that the center will in general be singular itself. Since a blowup of $X$ is induced by a blowup of the ambient space, the blowup $\widetilde{\mathbb{A}}^{3}$ of $\mathbb{A}^{3}$ with center $\operatorname{Sing}(X)$ might become itself singular. If we lose the smooth ambient space it is hard to measure the improvement of the singularities of $X$.

In the section on symmetry we will consider the surface Helix, which will be resolved in four different ways in order to illustrate the problem of finding an equivariant resolution.

Simplicity (mousse au chocolat). We consider the least involved type of point singularities a surface can have, namely simple singularities. Loosely speaking, an isolated singularity is simple if it can only be deformed into finitely many other types of isolated singularities. There are amazing connections between simple singularities and, e.g., real symmetry groups and quotient singularities. The beautiful paper of Durfee [6] lists fifteen different characterizations of simple singularities. Just as 
an appetizer of the various connections, if we start with equations, then we find Arnol'd's [1] complete classification of analytic functions $f:\left(\mathbb{C}^{3}, 0\right) \rightarrow(\mathbb{C}, 0)$ such that the singularity $(V(f), 0)=\left(f^{-1}(0), 0\right)$ is simple (the classification is up to local analytic isomorphism). These functions $f$ are polynomials of order 2 at 0 and are called $A D E$-functions; see [6. Table 1].

For us an interesting aspect of simple singularities is their resolution. If a surface has an isolated singularity that can be resolved by a sequence of point blowups, then this singularity is called absolutely isolated. Kirby 22] has shown that an isolated singularity $p$ of $X$ is simple if and only if it is absolutely isolated and the defining equation is of order 2 at $p$.

A simple singularity of a surface can also be characterized by data obtained from its resolution, i.e., by its dual resolution graph (or Dynkin diagram); see 6, Table 1]. The vertices of the dual resolution graph correspond to the exceptional curves on the so-called minimal resolution $\widetilde{X}$ of $X$. Two vertices are connected if the respective exceptional curves intersect on $\widetilde{X}$. The vertices are labeled with the self-intersection numbers of the respective exceptional curves.

Singularity (digestif). Until now we have tried to resolve surface singularities. In this section we look at the "inverse" problem of the construction of singularities. We try to find equations for surfaces with a prescribed singular locus. It is interesting to study surfaces with singular curves as a singular loci. In particular the construction of such a surface can probably give us hints concerning its resolution. Since we know how to improve singularities (blowup) we can use the inverse process (blowdown) to produce singularities. A blowdown will be a map that contracts a hypersurface. However, this procedure does not apply in a straightforward manner because an arbitrary blowdown of a surface will not lead to the desired singularity. The choice of the correct contraction is very subtle.

Example 1. We want a surface to have the plane Cusp $\left\{y^{2}=x^{3}, z=0\right\}$ as a singular locus. We try a straightforward method: suppose that $X$ is a surface in $\mathbb{A}^{3}$ with the Cusp in the $x y$-plane as a singular locus and whose equation we are searching for. Take this Cusp as the center of a blowup of $X$, with the reduced ideal $\left(x^{3}-y^{2}, z\right)$. A computation shows that in one chart the blowup $\widetilde{\mathbb{A}}^{3}$ of $\mathbb{A}^{3}$ is singular but in the other one $\widetilde{\mathbb{A}}^{3}$ is smooth. In the latter chart the corresponding affine blow-up map is

$$
\pi: \mathbb{A}^{3} \rightarrow \mathbb{A}^{3},(x, y, z) \mapsto\left(x, y,\left(x^{3}-y^{2}\right) z\right) .
$$

Restricting $\pi$ over $X$, we obtain the affine chart expression of the strict transform $X^{\prime}$ together with the blow-up map $\pi: X^{\prime} \rightarrow X$. We neither know equations of $X^{\prime}$ or $X$. Here $X^{\prime}$ can be any surface that is projected under $\pi$ to a surface with the Cusp as a singular locus. We may and will assume that $X^{\prime}$ is of a simple nature; for example, we take $X^{\prime}$ to be the cone given by the equation $x^{2}+y^{2}-z^{2}=0$. In order to get $X$ from $X^{\prime}$, apply the blow-down map

$$
\pi^{-1}: X \rightarrow X^{\prime},(x, y, z) \mapsto\left(x, y, \frac{z}{x^{3}-y^{2}}\right),
$$

which is well defined outside $X \cap V\left(x^{3}-y^{2}\right)$. Substituting $z \mapsto \frac{z}{x^{3}-y^{2}}$ in $X^{\prime}$ and multiplying with $\left(x^{3}-y^{2}\right)^{2}$ yields the equation for $X: z^{2}=\left(x^{2}+y^{2}\right)\left(x^{3}-y^{2}\right)^{2}$; see Figure 6, left. 
It is easy to see that the generic transversal section with a plane perpendicular to the singular locus of $X$, the Cusp, consists locally of two crossing lines. This means that $X$ has locally along its singular locus normal crossings. By replacing $z^{2}$ with $z^{3}$ we obtain the surface $X: z^{3}=\left(x^{2}+y^{2}\right)\left(x^{3}-y^{2}\right)^{2}$, and the generic transversal section becomes an irreducible singular curve. The result is shown in Figure 6, right.
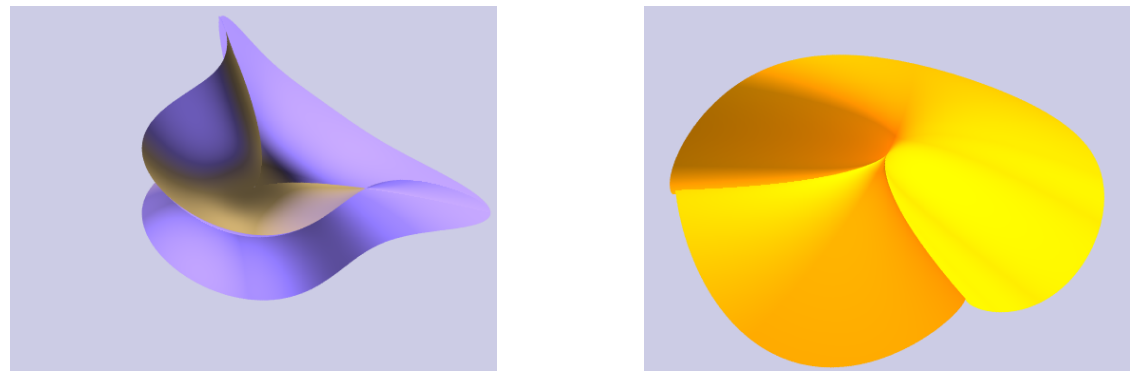

Figure 6. Surfaces with Cusp as singular locus.

Now the dinner starts. Enjoy your meal!

$$
\text { Triviality: Whitney umbrella } x^{2}=y^{2} z
$$

Our menu starts with a cold soup, a Gazpacho. The sample surface is the Whitney Umbrella. This is a singular surface $X$ in $\mathbb{A}^{3}$, defined by the equation $x^{2}-y^{2} z=0$; see Figure 7. It is also called a pinch point singularity.

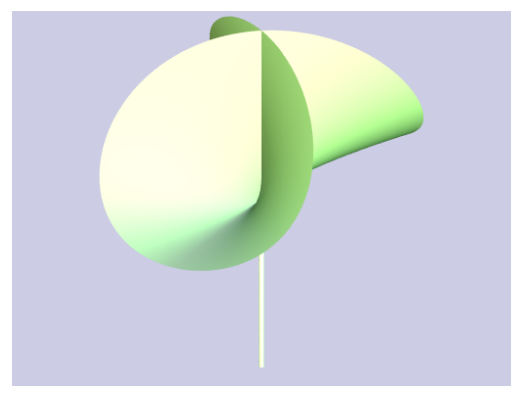

Figure 7. The Whitney Umbrella.

Let us describe its geometry in $\mathbb{A}_{\mathbb{R}}^{3}$. The Whitney Umbrella seems to consist of two components, namely the $z$-axis and a plane that is bent around the positive part of the $z$-axis in such a way that it intersects itself. As the defining polynomial $f=x^{2}-y^{2} z$ is prime in $\mathbb{R}[x, y, z]$ the surface $X$ is irreducible. The fact that $X$ looks like the union of a two-dimensional and a one-dimensional component arises from the visualization in real space: at each point in $\mathbb{A}_{\mathbb{R}}^{3}$ with negative $z$-coordinate the equation $f=0$ has just one solution, a point on the $z$-axis.

At points outside the $z$-axis the Whitney Umbrella is smooth and hence a manifold. The geometry along the singular locus $\operatorname{Sing}(X)$, the $z$-axis, is clearly more interesting. In Figure 7 one observes that in $\mathbb{A}_{\mathbb{R}}^{3}$ the singular locus is split into three 
different parts: at points with positive $z$-coordinate, $X$ looks locally like the union of two planes, and if $z<0$, then the Whitney Umbrella is locally one-dimensional. Only at the origin is the type of the singularity more involved.

We try to detect these local geometric properties by constructing a decomposition of $\operatorname{Sing}(X)$ into (locally closed) algebraic subsets along which $X$ looks locally the same. In the following we consider the Whitney Umbrella over the complex numbers, where it looks locally the same (again like two transversal planes) along the whole $z$-axis minus the origin.

The Euclidean topology together with analytic functions defined on open neighborhoods of points provides more flexibility regarding isomorphisms than the Zariski topology and regular functions on Zariski-open sets. We therefore view our algebraic surface $X$ for the moment as a complex analytic variety in $\mathbb{C}^{3}$. We say that $X$ is locally analytically isomorphic at two points $p$ and $q$ if there is a (Euclidean) neighborhood $U$ of $p$ in $\mathbb{C}^{3}$ and an analytic isomorphism $\varphi: U \rightarrow V$ onto an open neighborhood $V$ of $q$ sending $U \cap X$ onto $V \cap X$. In the language of germs, this is denoted by $(X, p) \cong(X, q)$. For fixed $p$ we call

$$
\operatorname{Triv}_{p} X=\{q \in X:(X, q) \cong(X, p)\}
$$

the trivial locus of $X$ at $p$. By a theorem of Ephraim [8, Thm. 0.2], Triv $p_{p} X$ is a submanifold of $X$ and the germ $(X, p)$ is isomorphic to the Cartesian product $\left(\operatorname{Triv}_{p} X, p\right) \times(Z, r)$ for some germ of an analytic variety $(Z, r)$. We then say that $X$ is locally at $p$ (analytically) trivial along $\operatorname{Triv}_{p} X$. It can happen that $\operatorname{Triv}_{p} X$ is reduced to the point $p$. This happens for instance if $X$ has an isolated singularity at $p$. In contrast, if $X$ is smooth, $\operatorname{Triv}_{p} X$ coincides with $X$ for all $p 1$

Let us now investigate the Whitney Umbrella with respect to this concept of local triviality. In any point $p=\left(p_{1}, p_{2}, p_{3}\right)$ of $X$ the germ of $X$ in $p$ is isomorphic to the germ $\left(V\left(f_{p}\right), 0\right)$ at 0 , where $V\left(f_{p}\right)$ denotes the zeroset of $f_{p}=f\left(x+p_{1}, y+p_{2}, z+p_{3}\right)$, which is just the Taylor expansion of $f$ in $p$. The singular locus of $X$ is the whole $z$-axis. To determine whether $X$ is analytically trivial along this axis we use the following Characterization of Local Analytic Triviality [4, Thm. 9.1.7]:

A family $\left\{\left(Y_{t}, 0\right)\right\}_{t \in \mathbb{C}} \subseteq\left(\mathbb{C}^{n}, 0\right)$ of germs of analytic varieties parametrized analytically by $t \in \mathbb{C}$ with $Y_{t}=V\left(g_{t}\right)$, defined by some analytic function $g_{t}=$ $g\left(x_{1}, \ldots, x_{n}, t\right)$ in $x_{1}, \ldots, x_{n}$ and $t$, is trivial at $t=0$ if and only if the derivative of $g_{t}$ with respect to $t$ lies in the ideal of the convergent power series ring $\mathbb{C}\{x, t\}$ generated by $g_{t}$ and the product of the maximal ideal with the Jacobian ideal,

$$
\partial_{t} g_{t} \in\left(g_{t}\right)+\left(x_{1}, \ldots, x_{n}\right)\left(\partial_{x_{1}} g_{t}, \ldots, \partial_{x_{n}} g_{t}\right) .
$$

This criterion plays an important role in the classification of isolated hypersurface singularities. It can also be used to prove the theorem of Mather-Yau and GaffneyHauser [30, 16, 2

Let us apply the criterion to the Whitney Umbrella $X$. Consider the family $\left\{\left(X_{t}, 0\right)\right\}_{t \in \mathbb{C}}$, where $X_{t}=V\left(f_{t}\right)$ and $f_{t}=f(x, y, z+t)=x^{2}-y^{2}(z+t)$ corresponds to the germ of $X$ in a point $(0,0, t)$ of the $z$-axis. A computation shows that $\partial_{t} f_{t}$ is not contained in the ideal $\left(f_{t}\right)+(x, y, z)\left(\partial_{x} f_{t}, \partial_{y} f_{t}, \partial_{z} f_{t}\right)$. Hence the Whitney

\footnotetext{
1 The same type of question occurs when considering an analytic family of germs $\left(X_{t}, p_{t}\right)$ parametrized by some space germ $(T, 0)$. In this case one asks for the locus of points $t$ where $\left(X_{t}, p_{t}\right)$ is isomorphic to $\left(X_{0}, p_{0}\right)$. Then the analogous result holds again; see [17.

${ }^{2}$ This theorem states that two analytic hypersurface germs are isomorphic if and only if their Tjurina algebras (i.e., the quotient of $\mathbb{C}\{x\}$ by the equation and the Jacobian ideal) are isomorphic.
} 
Umbrella is not locally at 0 analytically trivial along the $z$-axis. This corresponds to our impression from the real picture; cf. Figure 7.

Let us determine the trivial locus of $X$ at other points along the $z$-axis: in the point $(0,0,1)$ we have $\left(X_{1}, 0\right)=\left(V\left(f_{1}\right), 0\right)$ with $f_{1}=x^{2}-y^{2} z-y^{2}$. It is easy to see (cf. [13, I, example 5.3.6]) that $\left(X_{1}, 0\right)$ is locally isomorphic to the germ of $V\left(x^{2}-y^{2}\right)=V(x+y) \cup V(x-y)$, two transversal planes, at 0 . If $t \neq 0$, one can use the analytic isomorphism $\varphi_{t}: X_{t} \rightarrow X_{1},(x, y, z) \mapsto\left(x, \sqrt{t} y, \frac{z}{t}\right)$ to establish $\left(X_{1}, 0\right) \cong\left(X_{t}, 0\right)$. This shows that the trivial locus is the whole $z$-axis minus the origin. We have locally obtained at $p=(0,0, t)$ with $t \neq 0$ a decomposition of $X$ into a Cartesian product, namely

$$
(X, p) \cong(V(x, y), p) \times\left(V\left(x^{2}-y^{2}, z\right), 0\right) .
$$

We conclude that the origin is the most singular point of $X$.

In the algebraic category and taking biregular isomorphisms, the same type of questions is considerably more difficult:

Problem 1. Let $X$ be an algebraic variety over a field $K$. Let $p$ be a fixed point on $X$. Consider $\operatorname{Triv}_{p} X=\{q \in X:(X, q) \cong(X, p)\}$, where $(X, p)$ now denotes the (algebraic) germ of $X$ in $p$ with respect to the Zariski topology and $\cong$ means biregularly isomorphic. Is $\operatorname{Triv}_{p} X$ a (smooth) algebraic subset of $X$ ? Is there a local decomposition $(X, p)=\left(\operatorname{Triv}_{p} X, p\right) \times Y$ for some algebraic germ $Y$ ?

Let us now discuss the resolution of the Whitney Umbrella $X$ by blowups. The pictures will again be in $\mathbb{A}_{\mathbb{R}}^{3}$, though our reasoning is mostly based on the complex setting. At the beginning we need to choose the locus of points where $X$ will be modified, i.e., the center of the first blowup. As a blowup $\pi: \widetilde{X} \rightarrow X$ is an isomorphism outside its center $Z$ and we do not want to alter the smooth points of our variety, we require that $Z$ lies inside the singular locus $\operatorname{Sing}(X)$. Furthermore, we prefer to have a smooth center $Z$. Blowups in singular centers are not well understood because they may introduce singularities in originally smooth varieties (e.g., in $\mathbb{A}^{3}$ ). In the section Symmetry this topic is raised with more detail.

Since $\operatorname{Sing}(X)$ is in our case just the $z$-axis, the only smooth subvarieties of Sing $(X)$, and hence possible centers, are points on the $z$-axis given by ideals $(x, y, z-t)$, where $t \in K$, or the whole axis, given by the ideal $(x, y)$. In the preceding discussion we have seen that the origin is the most singular point of the surface. So we try the origin $Z=V(x, y, z)$ as the center of the blow up. Let $\pi: \widetilde{\mathbb{A}}^{3} \rightarrow \mathbb{A}^{3}$ be the blow-up map of the ambient space with center $Z$ (by abuse of notation we will sometimes also denote the ambient blow-up map with $\pi$ ). The blowup $\widetilde{\mathbb{A}}^{3}$ of $\mathbb{A}^{3}$ is covered by three affine charts. The corresponding blow-up maps are

$$
\pi_{x}: \mathbb{A}^{3} \longrightarrow \mathbb{A}^{3},(x, y, z) \mapsto(x, x y, x z),
$$

resp.

and

$$
\pi_{y}: \mathbb{A}^{3} \longrightarrow \mathbb{A}^{3},(x, y, z) \mapsto(x y, y, y z)
$$

$$
\pi_{z}: \mathbb{A}^{3} \longrightarrow \mathbb{A}^{3},(x, y, z) \mapsto(x z, y z, z) .
$$

We shall see that in the $x$-chart the singularity has disappeared and that it has "improved" in the $y$-chart whereas the singularity in the $z$-chart has remained the same. In the $x$-chart we get the total transform $X^{*}=V\left(f^{*}\right)$, where $f^{*}=$ $x^{2}\left(1-x y^{2} z\right)$. The strict transform $f^{\prime}=1-x y^{2} z$ is smooth and does not intersect 
the exceptional component, which is the plane $x=0$. Thus the singularity of $X$ is resolved in the $x$-chart; cf. Figure 8

In the $y$-chart the strict transform $f^{\prime}=x^{2}-y z$ defines a cone with vertex at the origin. Clearly the singularity has improved along the $z$-axis because all points not equal to the origin have become smooth in the $y$-chart. However, the origin is still singular. So we ask if this singularity at the origin has somehow improved. There is no unique way to answer this question. The most natural idea is to use the order of the defining ideal in each point of $X$ as a measure: for a singular point the order is strictly greater than one and if the order has dropped to one the point has become smooth. In our case the order has remained constant at the origin, so one has to consider a finer measure. We will not go into detail but with the help of resolution invariants one can detect an improvement of this singularity; for details see [2, 3, 15]. Now there is just one chart missing, the $z$-chart. This is the critical chart, where our attempt of a resolution will come to an abrupt end. The strict transform is $f^{\prime}=x^{2}-y^{2} z$. Then $f=f^{\prime}$, that is, $X^{\prime}$ has the same singularity as $X$ in this chart. So there is no way to declare $X^{\prime}$ less singular than $X$. We conclude that the center of the blowup was too small to resolve or to improve the singularity of $X$.
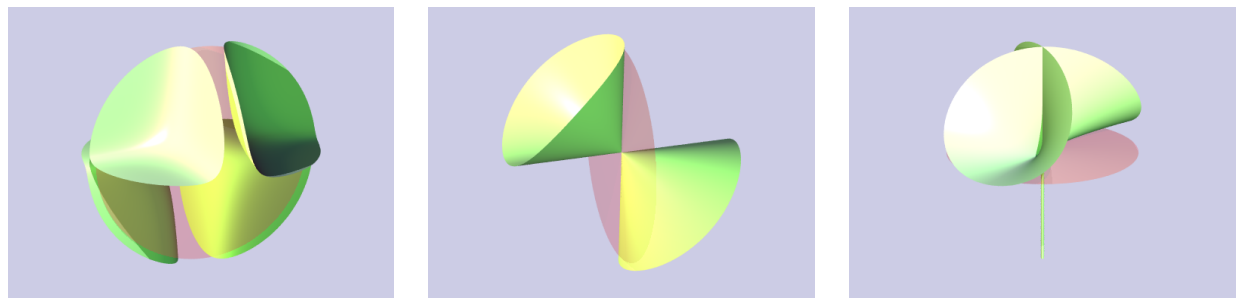

FiguRE 8 . The three charts of the point blowup ( $x$-chart, $y$-chart and $z$-chart, exceptional divisor in red).

The origin was geometrically a reasonable center, as we saw with the decomposition of $X$ into locally trivial strata. But this decomposition turns out to be misleading for resolution purposes. Instead, we use the stratification induced by the local order $\operatorname{ord}_{p}(f)$ of the defining function. It is the order of the Taylor expansion of $f$ in $p$. Denote top $(f)=\left\{p \in \mathbb{A}^{3}, \operatorname{ord}_{p}(f)\right.$ is maximal $\}$, the top locus of $f$. Choosing the center of the blowup in the top locus of $X$ is very suitable since then, as can be shown without much effort, the order does not increase under blowup. If it drops, one can apply induction; if it remains constant, a second invariant has to be defined in order to exhibit an improvement of the singularities.

For $f=x^{2}-y^{2} z$ the order of $f$ is 2 at all points of the $z$-axis and $f$ is of order 1 at any other point of $X$. Hence $\operatorname{top}(f)$ is the $z$-axis defined by the ideal $(x, y)$. Now consider the blowup of $\mathbb{A}^{3}$ with center the $z$-axis. This blowup is covered by two affine charts, corresponding to the two generators $x$ and $y$ of the ideal of the center: in the $x$-chart the total transform $f^{*}$ is defined by the equation $x^{2}\left(1-y^{2} z\right)=0$. The strict transform is the Cartesian product of a hyperbola and the $x$-axis and intersects the exceptional plane $V(x)$ in the hyperbola $V\left(x, 1-y^{2} z\right)$; see Figure 9 , In this figure the exceptional plane $V(x)$ is depicted in blue. The total transform $X^{*}$ has normal crossings. Therefore the singularity is resolved in this chart; see Figure 9 . 
In the $y$-chart the strict transform is $f^{\prime}=x^{2}-z$, the Cartesian product of a parabola in the $x z$-plane with the $y$-axis; see Figure 9. The strict transform is smooth and intersects the exceptional plane $V(y)$ transversally. The total transform $X^{*}$ has normal crossings in this chart; therefore we are done.

We have now resolved the Whitney Umbrella $X$ with a single blowup. The blowup $X^{\prime}$ lives in $\mathbb{A}^{3} \times \mathbb{P}^{1}$. To describe how the two charts patch together we compute the affine chart expressions of the blow-up map. This yields the transition maps between the two charts: from the $x$ - to the $y$-chart,

$$
\varphi_{x y}: X_{x}^{\prime} \longrightarrow X_{y}^{\prime},(x, y, z) \mapsto\left(\frac{1}{y}, x y, z\right),
$$

and from the $y$ - to the $x$-chart,

$$
\varphi_{y x}: X_{y}^{\prime} \longrightarrow X_{x}^{\prime},(x, y, z) \mapsto\left(x y, \frac{1}{x}, z\right) .
$$

Let $z$ go to infinity in both charts; see Figure 9. All lines parallel to the $x y$-plane in the $x$-chart stay parallel in the $y$-chart. In these pictures the blue resp. green parts in the $x$-chart have to be glued together with the corresponding blue resp. green parts in the $y$-chart. In Figure 10, a schematic picture of the result of the patching can be seen: As the transition maps suggest, the orientation of $X^{\prime}$ has to change between the two charts. This is indicated in Figure 10 by a Moebius band in the blue part. The blue rectangles in the picture indicate the exceptional planes.
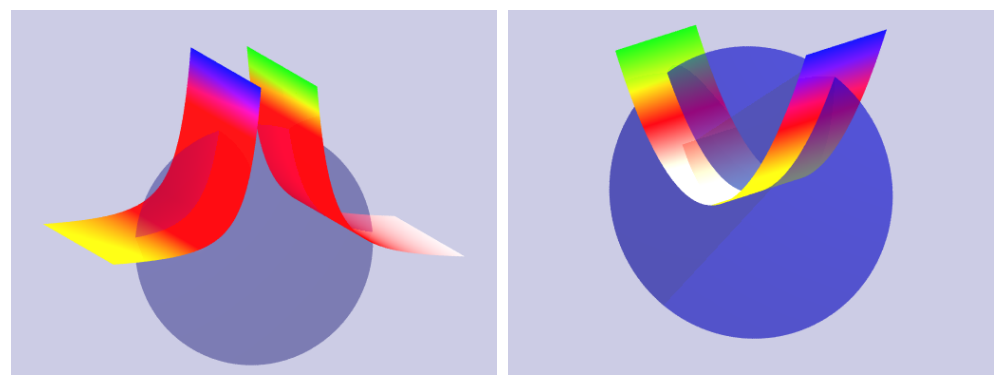

Figure 9. $x$-chart (left) and $y$-chart (right).

TANGENCY: Kolibri $y^{2}=x^{2} z^{2}+x^{3}$

Let us move on to the next course, a fresh salad. What kind of dressing would you like? In this course we study the surface Kolibri. Its singularities are perfectly suited for illustrating limits of tangent planes. The singular locus of Kolibri is a line, the $z$-axis, along which the singularity type is, outside the origin, that of two transversal planes. Only at the origin is the singularity more complicated. Recall the appetizers section where we constructed Kolibri: we took the plane Node with equation $y^{2}=x^{3}+x^{2}$ and placed it in three-space on a horizontal plane at height $z=1$. We moved this plane with the curve downwards while contracting the Node (keeping the intersection point on the $z$-axis) and got the equation of Kolibri

$$
K: y^{2}=x^{2} z^{2}+x^{3} .
$$




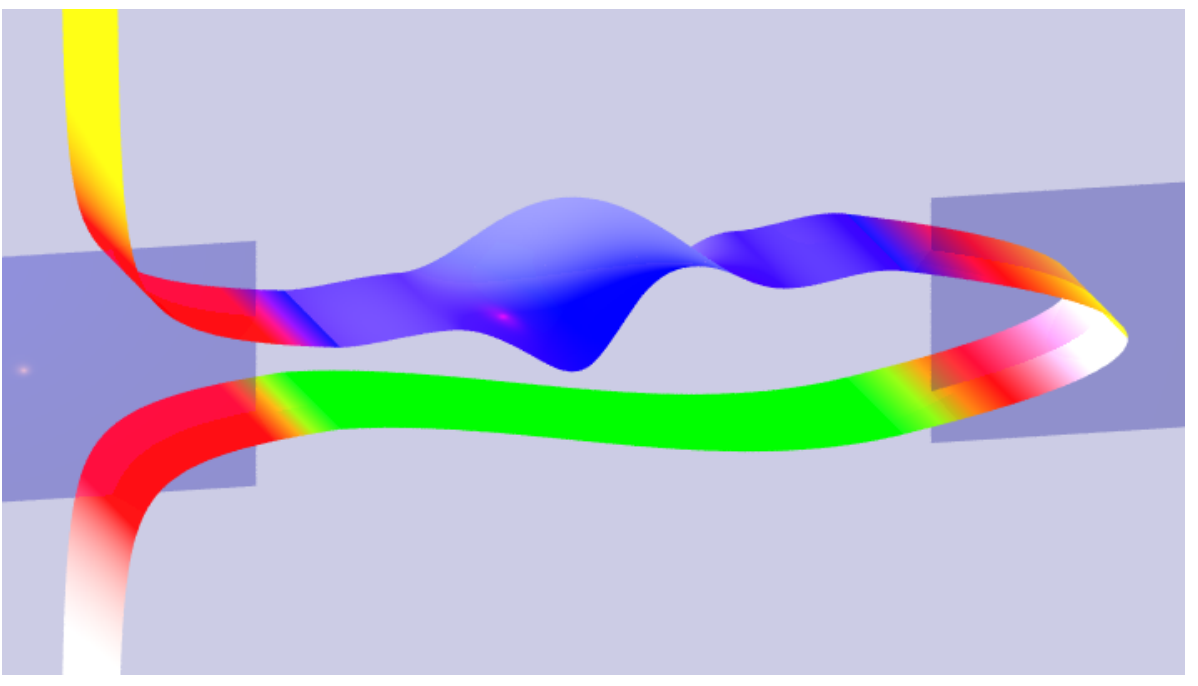

Figure 10. Patching the two charts of the blowup of the Whitney Umbrella.
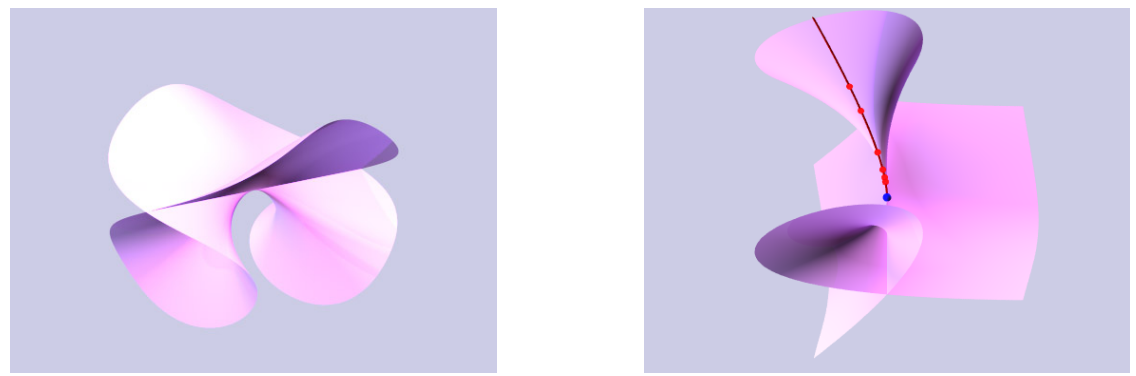

Figure 11. Kolibri flying and Whitney's (b) condition.

The plane section $z=0$ is the Cusp $x^{3}=y^{2}$, and the surface is symmetric with respect to $\pm z$; see Figure 11, right.

Let us now describe the limits of tangent planes as the tangency point (this is the base point of the tangent plane) moves from the smooth part into the singular locus $Y$ of our surface $K$. If the limit point in $Y$ is different from the origin, everything behaves as expected: the limiting plane contains the tangent line to the singular locus. This also holds if the sequence of points approaches the origin: let $p=(a, b, c) \in K$ be a smooth point close to the origin. The tangent plane is given by $T_{p}(K):\left(2 a c^{2}+3 a^{2}\right) x-2 b y+2 a^{2} c z=0$. Taking the limit as $p$ goes to 0 , the limiting tangent plane contains the $z$-axis. Therefore Whitney's condition (a) is satisfied at any point along $Y$ (see the appendix or [37, 38] for a detailed description of Whitney's conditions).

A finer notion of "fitting together" of the two parts of Kolibri, namely $K \backslash Y$ and $Y$, is obtained by considering limits of secants $\overline{x_{i} y_{i}}$ of sequences of points $x_{i}$ in $K \backslash Y$ and $y_{i}$ in $Y$, both approaching $y \in Y$. If the limit of these secants is contained in the limit of the tangent spaces of the $x_{i}$ for all possible sequences of $x_{i}$ and $y_{i}$ 
(for which the limit exists) the adjacent pair of strata $(K \backslash Y, Y)$ is said to satisfy Whitney's condition (b) at $y$.

Whitney's condition (b) holds at any point on the $z$-axis minus the origin. But if the sequence of points converges to the origin it is checked (consider the intersection of Kolibri with the plane defined by $y=0$ ) that there are limits of secants that are not contained in the limit of the respective tangent planes. Thus condition (b) does not hold at 0. In Figure 11 (right), a part of the intersection curve of Kolibri with the plane $y=0$ and a sequence of points violating Whitney's condition (b) are sketched. Condition (b) allows us to distinguish the origin from the remaining points of $Y$ as a singular point with higher complexity. This is expressed by saying that $K$ satisfies Whitney's condition (a), but not (b), along the stratum $Y$, locally at 0 .

Now we turn to the problem of resolving Kolibri. By the above considerations, the origin is the most singular point of our surface. If we choose it as the center of the blowup, the singularity does not improve (in the $z$-chart, the new equation is $y^{2}=x^{2} z^{2}+x^{3} z$ ). So, despite the reasonable strategy to try to get rid of the worst singularities first, this was not a suitable choice. We therefore apply a curve blowup with center the entire singular locus of $K$. Taking this line blowup, it can easily be seen what happens along the singular locus of $K$ at points outside the origin. As $K$ locally looks there like the union of two planes meeting transversally along the $z$-axis, the blowup separates these two local components. Moreover, both components meet the ambient exceptional divisor (recall: this is a "plane" in $\widetilde{\mathbb{A}}^{3}$ ) transversally, as long as we avoid the origin. So the interesting transformation happens above the origin. The fibre over 0 in the blowup $X^{\prime}$ does not meet the $y$-chart and consists of two transversal lines in the $x$-chart; see Figure 12 .
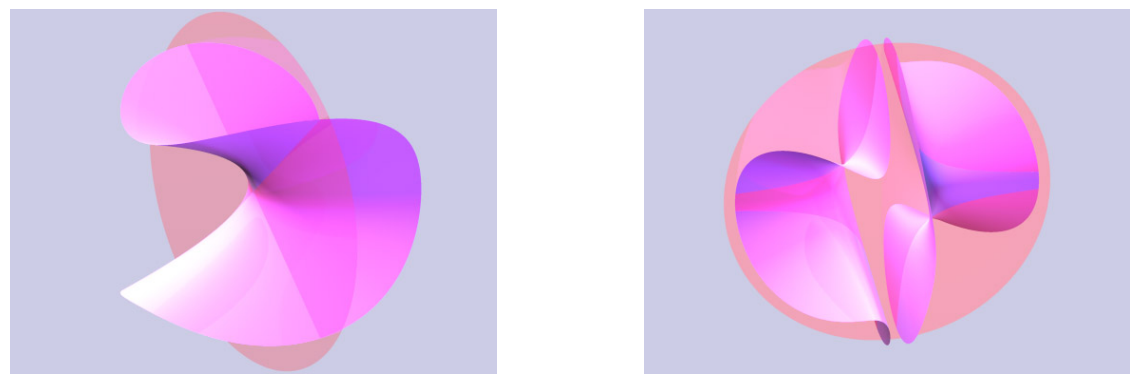

Figure 12. The $x$ - and $y$-chart of the blowup of the singular $z$-axis.

It is therefore sufficient to consider the $x$-chart of the blowup. A computation yields that in this affine chart the equation of the strict transform $K^{\prime}$ of $K$ is $x+z^{2}=y^{2}$, a saddle. It is smooth. The exceptional divisor $\mathbb{E}$ in $\widetilde{\mathbb{A}}^{3}$ is locally given as the plane $x=0$. The exceptional divisor $\mathbb{E} \cap K^{\prime}$ in $K^{\prime}$ consists of the $x$ chart of the two lines defined by $V\left(y^{2}-z^{2}, x\right)$. Since $\mathbb{E} \cup K^{\prime}$ does not have normal crossings, Kolibri is not yet resolved (recall that we want to achieve an embedded resolution). The origin of the $x$-chart is the only point where $\mathbb{E} \cup K^{\prime}$ does not have normal crossings; hence we use it as the center of the next blowup. This blowup will separate the two components of $\mathbb{E} \cap K^{\prime}$. After the blowup the total transform $\left(K^{*}\right)^{*}$ of $K^{\prime}$ consists of its strict transform $K^{\prime \prime}$, the transform of the old exceptional plane (depicted in red in Figure 13) and the new exceptional plane (depicted in green in 
Figure 13). The $x x$-chart can be discarded since here the strict transform is smooth and does not meet any of the two exceptional components.
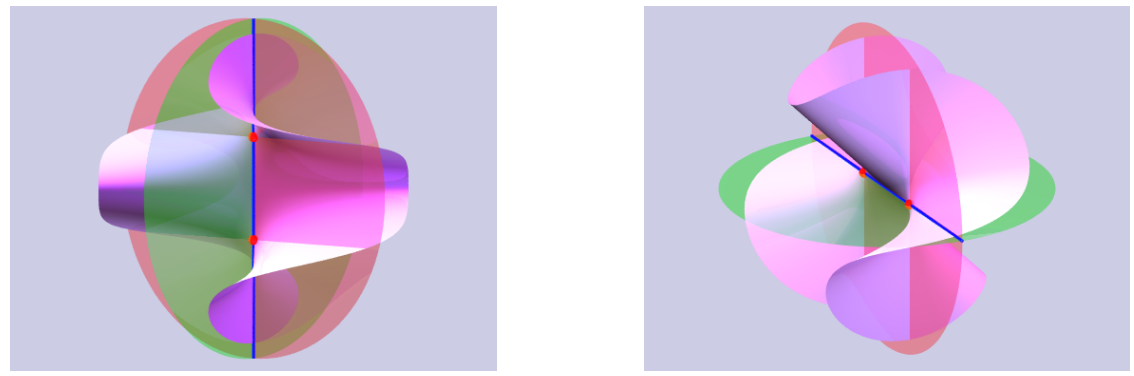

FIgURE 13 . The $x y$ - and the $x z$-chart of the point blowup of the saddle.

We now have three exceptional curves on $K^{\prime \prime}$, which are smooth and intersect transversally in two points marked in red in Figure 13. These two intersection points are contained in the $x y$ - and the $x z$-chart. Notice the symmetry of the $x y$ and the $x z$-chart via the coordinate transformation $(x, y, z) \mapsto(x,-z, y)$. However, we are not finished yet.

For our goal, an embedded resolution, the total transform of $K$ must have normal crossings everywhere. As one can see in Figure 13 the strict transform $K^{\prime \prime}$ meets the red exceptional plane tangentially in the intersection points mentioned above. If we just blow up the two points, we will not improve the situation, because in one chart we get the same strict transform as before. Therefore we choose a larger center: we blow up the line joining the two "bad" points (this line is contained in the strict transform $\left.K^{\prime \prime}\right)$. One can see in Figure 14 that in the resulting charts the total transform has everywhere normal crossings. Hence Kolibri is resolved.
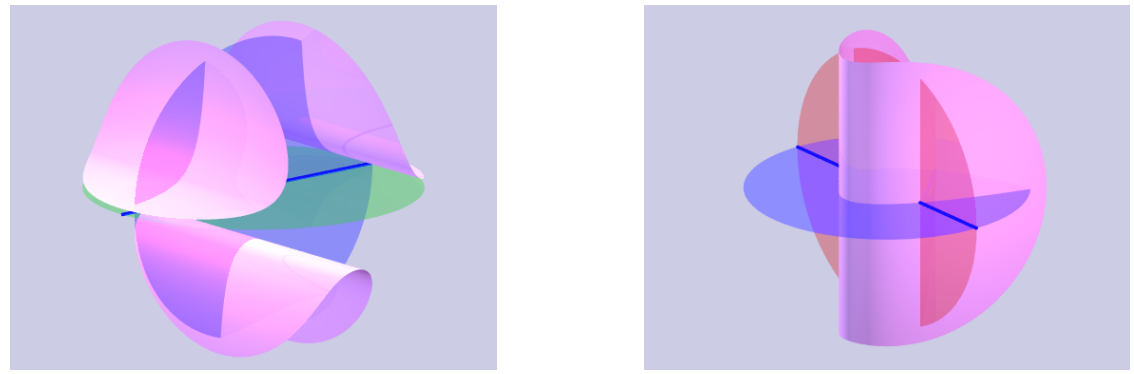

FiguRE 14. Normal crossings in the $x z x$ - and the $x z z$-chart after three blowups. 
In the diagram one can see the blowups that led to the resolution of Kolibri:

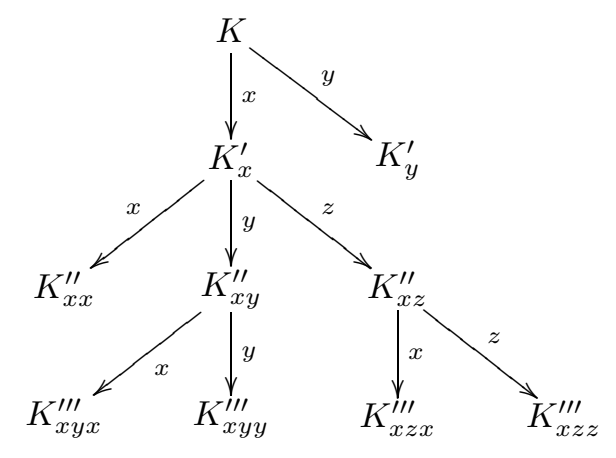

Transversality: IrIS, $x^{2} y+y^{2} z=z^{4}$

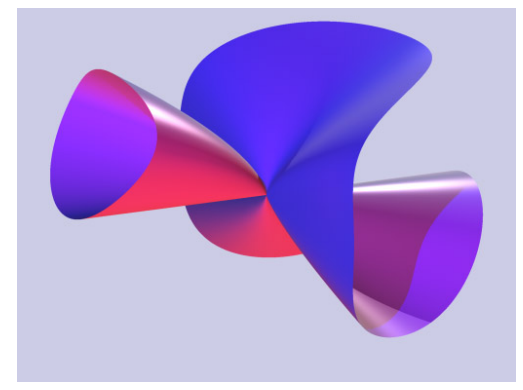

Figure 15. Iris, $x^{2} y+y^{2} z=z^{4}$.

Our menu continues with a plate of fish or seafood. We consider a surface called Iris, which looks like a blossom. It consists of a calyx and a leaf bent around it, connected just at one point; see Figure 15. This point, the origin, is the singular locus of Iris. The surface is irreducible; hence one cannot separate the two parts algebraically. However, if we consider a small Euclidean neighborhood of the origin, Iris looks locally like the union of the cone $x^{2}+y z=0$ with the plane $y=0$. One can resolve the cone by blowing up the origin, which leaves the plane $y=0$ unchanged. This observation suggests that a point blowup could already smooth Iris. Indeed, one point blowup will make the strict transform smooth; however, the total transform has not yet normal crossings. We have chosen Iris because its resolution led us to discuss various notions of transversality: clean intersection, normal crossings and mikado. We start with explaining these different concepts.

1. Clean intersection. Two smooth varieties $X, Y \subseteq \mathbb{A}^{n}$ defined by radical ideals $I_{X}$, resp. $I_{Y}$, intersect cleanly if their intersection $Z=X \cap Y=V\left(I_{X}+I_{Y}\right)$ is smooth and if the tangent spaces satisfy

$$
T_{p}(Z)=T_{p}(X) \cap T_{p}(Y) \text { for all } p \in Z \text {. }
$$

Equivalently, at all points of the intersection of $X$ and $Y$, there is a local analytic isomorphism of $\mathbb{A}^{n}$ mapping $X$ and $Y$ onto linear subspaces. This equivalence can be easily shown using the implicit function theorem. 
In [27, Fact 5.1], Li Li gives the following characterization of clean intersections: let $X, Y$ be smooth varieties in $\mathbb{A}^{n}$ defined by radical ideals $I_{X}, I_{Y}$. Then $X$ and $Y$ intersect cleanly if and only if their set-theoretical intersection $X \cap Y$ is smooth and the ideal $I_{X \cap Y}:=I_{X}+I_{Y}$ is radical. We then also say that the intersection $X \cap Y$ is scheme-theoretically smooth.

2. Normal crossings. The next notion is used to characterize intersections of several components. Let $X_{1}, \ldots, X_{k}$ in $\mathbb{A}^{n}$ be smooth varieties. Then $X_{1}, \ldots, X_{k}$ have normal crossings at $p$ if there exists a local system of coordinates around $p$ such that each $X_{i}$ is defined by the vanishing of some of the coordinates. We say that $X_{1}, \ldots, X_{k}$ have normal crossings in $\mathbb{A}^{n}$ if they have normal crossings at any of their points.

We say that a variety $X$ has normal crossings or that $X$ is a normal crossings variety if its irreducible components $X_{1}, \ldots, X_{k}$ have normal crossings. Let $Y$ be another normal crossings variety with irreducible components $Y_{1}, \ldots Y_{l}$. We say that $X$ is transversal to $Y$ if $X_{1}, \ldots, X_{k}, Y_{1}, \ldots, Y_{l}$ have normal crossings.

3. Mikado. Smooth subvarieties $X_{1}, \ldots, X_{k}$ of $\mathbb{A}^{n}$ are mikado if all possible intersections

$$
\bigcap_{j \in J} X_{j} \quad \text { with } J \subseteq\{1, \ldots, k\}
$$

are scheme-theoretically smooth. If $X_{1}, \ldots, X_{k}$ have normal crossings, then they are mikado. For two surfaces in $\mathbb{A}^{3}$, mikado and clean intersection are equivalent.

Let us now see how these notions appear in a resolution process of a variety. In the case of Iris we will find that after the first blowup (with center the origin) the strict transform is smooth. The total transform is singular since it is the union of the strict transform and the exceptional plane $\mathbb{E}$. Both intersect in a curve. We see that $X$ is resolved (in the embedded sense) if and only if $X^{\prime}$ is transversal to the exceptional locus $\mathbb{E}$. After $k$ blowups the total transform $X^{*}$ of a surface $X$ will consist of $k$ exceptional surfaces plus the strict transform $X^{(k)}$ of $X$.

Let $X \subset \mathbb{A}^{3}$ be a surface having normal crossings and let $Y$ be a variety transversal to $X$. If we blow up in a smooth center transversal to $X$ and contained in $X \cap Y$, then the blowup of $X$ will again have normal crossings and the strict transform of $Z$ will have a clean intersection with the exceptional divisor. However, achieving normal crossings can be a lengthy and tedious procedure. Often it is convenient to aim only at mikado singularities. But this notion need not be stable under blowup 3

Therefore we pose the following

Problem 2. Let $Y=\left(Y_{1}, \ldots, Y_{k}\right)$ be mikado in $\mathbb{A}^{n}$. Characterize all subvarieties $Z \subseteq Y$ such that the blowup of $\mathbb{A}^{n}$ in $Z$ transforms $Y$ again into mikado.

\footnotetext{
${ }^{3}$ Example 2 (communicated by $\mathrm{Li} \mathrm{Li}$ ). Let $M$ be the union of the $z$-axis $l$, the $x y$-plane $P$ and the large diagonal $x=z$, denoted by $Q$, in $\mathbb{A}^{3}$. Then $M$ is defined by the product ideal $(x, y)(z)(z-x)=(x z(z-x), y z(z-x))$. We see that $M$ is mikado. Consider the blowup of $\mathbb{A}^{3}$ with center the $z$-axis. We get two affine charts (corresponding to the two generators of the center). In the $y$-chart the total transform $M^{*}$ is defined by the ideal $(x y z(z-x y), y z(z-x y))$. We see that the strict transform $P^{\prime}$ of $P$ remains unchanged but $Q$ is transformed into the saddle $Q^{\prime}=V(z-x y)$. The line $l$ becomes the exceptional component $\mathbb{E}=V(y)$. However, $M^{*}=P^{\prime} \cup Q^{\prime} \cup E$ is not mikado since $P^{\prime} \cap Q^{\prime}=(z, x y)$ is the union of two intersecting lines and is thus singular.
} 
We now resolve Iris in three blowups. Below is a table in which one can see how the resolution works. We denote Iris by $T: x^{2} y+y^{2} z-z^{4}=0$.

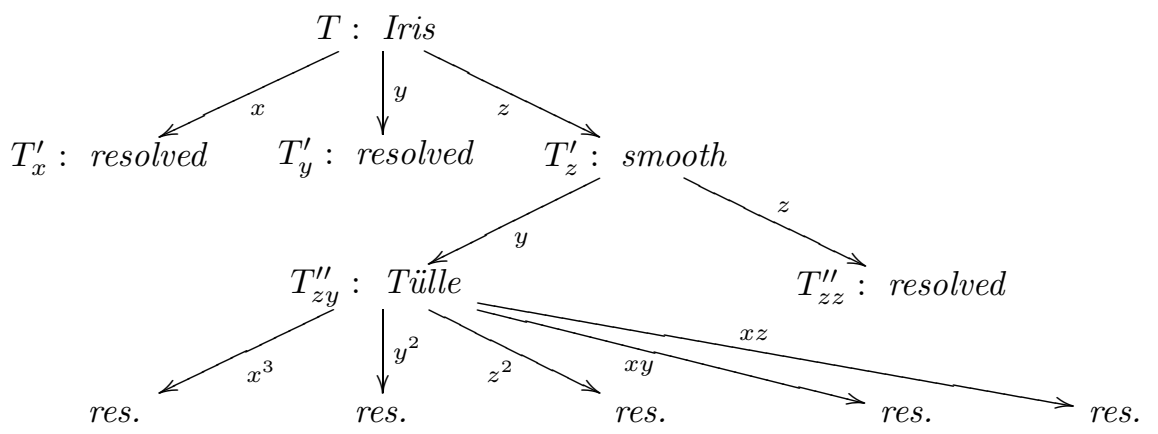

The first blowup with center the origin produces a smooth strict transform $T^{\prime}$. In the $x$ - and $y$-chart one can easily see that the exceptional divisors and the strict transform have normal crossings; see Figure 16, left and middle. However, in the $z$-chart, the strict transform $x^{2} y+y^{2}=z$ and the exceptional divisor do not have normal crossings since the intersection curve is singular at the origin.
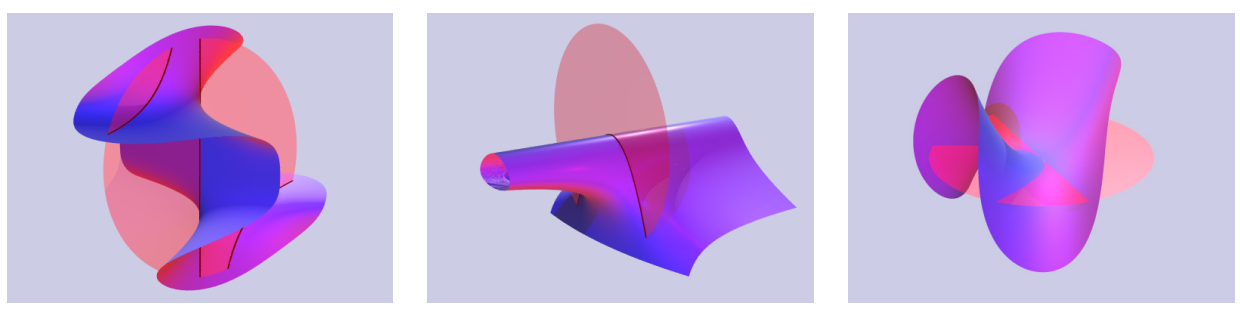

FiguRE 16. The three affine charts of the point blowup of Iris.

We have to perform another blowup. We could again choose the origin of the $z$ chart as center but a computation shows that things do not improve. For example, in the $z x$-chart the strict transform and the exceptional component $V(z)$ do not meet transversally.

In order to improve the situation we choose a larger center, for instance the $x$-axis in the $z$-chart. This axis is a component of the intersection $T_{z}^{\prime} \cap \mathbb{E}$. The blowup is covered by two affine charts. In the $z z$-chart the strict transform $T_{z z}^{\prime \prime}$ is smooth and meets the exceptional component $V(z)$ transversally; see Figure 17. In the $z y$-chart the total transform $T_{z y}^{*}=V\left(y z\left(x^{2}+y-z\right)\right)$ is the union $V(y) \cup V(z) \cup V\left(x^{2}+y-z\right)$. This surface is called Tülle; see Figure 17.

For Tülle, all pairwise intersections of the three components are smooth: the $x$-axis is the intersection of the two exceptional surfaces, and $T_{y z}^{\prime \prime}$ defined by the equation $x^{2}+y=z$ intersects each exceptional component in a parabola. But the three intersection curves meet tangentially at the origin. Said differently, the intersection of the three components of Tülle is the origin with non-reduced ideal $\left(x^{2}, y, z\right)$, which is not radical. Hence Tülle is not mikado (it is the simplest such example).

In order to obtain normal crossings we could blow up the origin of Tülle again since it is the only point that is an obstacle to being mikado. A computation shows that this blowup would indeed yield mikado but still no normal crossings for the 

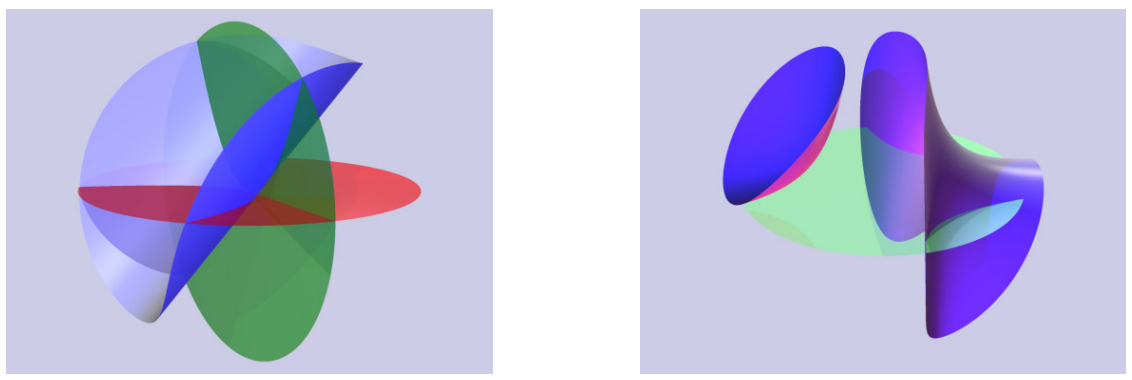

Figure 17. Second blowup of Iris: $z y$-chart Tülle (left), $z z$-chart (right).

total transform. So we try to blow up the nonreduced ideal $\left(x^{2}, y, z\right)$, which defines the common intersection of the three components of Tülle. Blowing up this ideal yields a singularity in the ambient space $\widetilde{\mathbb{A}}^{3}$. As with the cross for Helix, see the section Symmetry, we use an ideal whose associated blowup leaves the ambient space smooth. The ideal that works is $\left(x^{2}, y, z\right)(x, y, z)=\left(x^{3}, y^{2}, z^{2}, x y, x z\right)$. The resulting blowup $\widetilde{\mathbb{A}}^{3}$ is isomorphic to the blowup of the origin in $\mathbb{A}^{3}$ followed by the blowup of the origin of the hereby obtained $x$-chart (just compute the chart expressions).

In the $x y$-chart and the $x^{3}$-chart the total transform of Tülle is a union of four planes, whose pairwise and triple intersections are smooth and whose quadruple intersection is empty. It has normal crossings and hence is also mikado. In the $y^{2}$ chart the strict transform is a saddle-surface defined by the equation $x^{2} y+1=z$. Moreover the total transform $T_{z y\left(y^{2}\right)}^{*}$ is the union of the saddle and two coordinate planes; see Figure 18. The common intersection of its three components is empty. An easy computation shows that $T_{z y\left(y^{2}\right)}^{*}$ has normal crossings. The expressions of the total transforms in the two remaining charts are similar to the $x y$ - resp. $y^{2}$ chart, and one can go from one to the other by permuting $y$ with $z$. Hence we are also finished in these two charts.

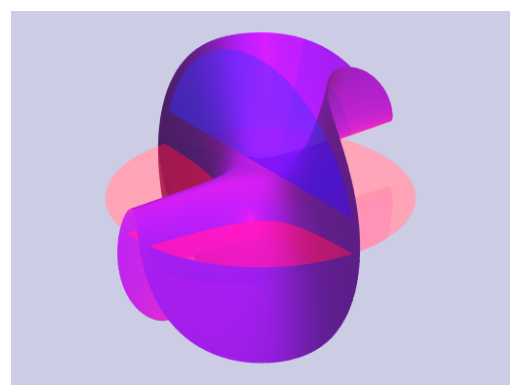

FiguRE 18. The $y^{2}$-chart of the third blowup of Iris.

$$
\text { Symmetry: Helix } x^{4}+y^{2} z^{2}=x^{2}
$$

In the second main course, a roast, we study the surface Helix. It fascinates us by its twofold symmetry: four petals emerge from the positive and negative $y$ - and 
$z$-axes. Along these axes a transversal plane section exhibits the figure eight curve Lemniscate (see Figure 19).

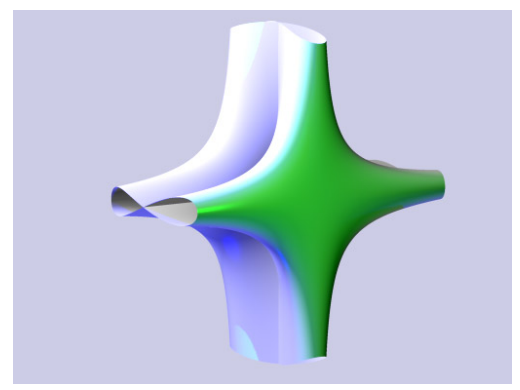

Figure 19. Helix.

The singular locus of Helix is a cross, namely the two axes in the $y$ - and $z$ direction, which is formed as the intersection of two smooth transversal sheets as long as we stay outside the origin. At the origin the geometry is more involved because on each of the four rays the two sheets of the surface approach each other (see Figure 20).

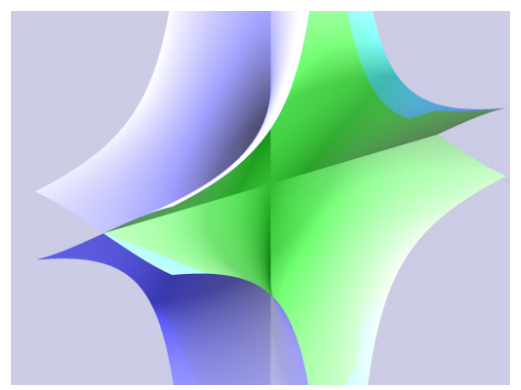

Figure 20. Helix sliced open near the origin.

If one only looks at the real part of Helix the $x$-coordinate of the points on the surface remains bounded by 1 . The variable $x$ can be expressed as a double root

$$
x= \pm \frac{1}{2} \sqrt{2 \pm 2 \sqrt{1-4 y^{2} z^{2}}} .
$$

In the description of Helix (the name comes from the Greek $\epsilon \iota \lambda \epsilon \iota \nu$ : to turn, rotate) the word symmetry has already appeared. We now describe particular symmetries of Helix and study how they are related to the construction of this surface. Looking at the real picture (Figure 19) one immediately sees that one can rotate the surface 90,180 and 270 degrees around the $x$-axis, or reflect it with respect to the $x y$-, $y z$ or $x z$-plane without changing its shape and position in $\mathbb{A}^{3}$. These automorphisms are contained in the symmetry group of the Helix.

The symmetry group $\operatorname{Aut}(Y)$ of an algebraic variety $Y \subseteq \mathbb{A}^{n}$ is the group of all polynomial automorphisms of $\mathbb{A}^{n}$ that fix $Y$, i.e., $\operatorname{Aut}(Y)=\left\{\varphi \in \operatorname{Aut}\left(\mathbb{A}^{n}\right): \varphi(Y)=\right.$ $Y\}$. Note that for $Y=V(g)$ a hypersurface, $Y$ is invariant under the action of a group $G \subseteq \operatorname{Aut}\left(\mathbb{A}^{n}\right)$ if $g\left(x_{1}, \ldots, x_{n}\right)=\lambda g\left(\varphi^{-1}\left(x_{1}, \ldots, x_{n}\right)\right)$ for every $\varphi \in G$ and 
some $\lambda=\lambda_{\varphi} \in K^{*}$. Of course one can test if the surface $Y$ is invariant under a specific group $G \subseteq \operatorname{Aut}\left(\mathbb{A}_{\mathbb{R}}^{3}\right)$, but there seem to be no general results. However, the computation of $\operatorname{Aut}(Y)$ is not straightforward since the group $\operatorname{Aut}\left(\mathbb{A}_{K}^{n}\right)$ for $n \geq 2$ is hard to understand 4

Let $X$ denote Helix viewed in $\mathbb{A}_{\mathbb{R}}^{3}$. Since the group $\operatorname{Aut}(X)$ of polynomial automorphisms is rather complicated we will look at the group of linear symmetries of Helix

$$
\operatorname{Aut}_{\text {lin }}(X)=\{\varphi \in \operatorname{Aut}(X): \varphi \text { linear }\} .
$$

Its elements can be represented by $3 \times 3$ matrices. This group is generated by the matrices (here all signs are independent)

$$
\left(\begin{array}{ccc} 
\pm 1 & 0 & 0 \\
0 & 1 & 0 \\
0 & 0 & 1
\end{array}\right),\left(\begin{array}{ccc}
1 & 0 & 0 \\
0 & \pm \lambda & 0 \\
0 & 0 & \pm \frac{1}{\lambda}
\end{array}\right),\left(\begin{array}{ccc}
1 & 0 & 0 \\
0 & 0 & \pm \lambda \\
0 & \pm \frac{1}{\lambda} & 0
\end{array}\right), \lambda \in \mathbb{R}^{*} .
$$

It is isomorphic to the group $\mathbb{Z}_{2} \times \mathcal{G}$, where $\mathcal{G}$ is generated by the $2 \times 2$ matrices

$$
M_{0}(\lambda)=\left(\begin{array}{cc}
\lambda & 0 \\
0 & \frac{1}{\lambda}
\end{array}\right), M_{1}(\lambda)=\left(\begin{array}{cc}
\lambda & 0 \\
0 & -\frac{1}{\lambda}
\end{array}\right), M_{2}(\lambda)=\left(\begin{array}{cc}
0 & \lambda \\
\frac{1}{\lambda} & 0
\end{array}\right), M_{3}(\lambda)=\left(\begin{array}{cc}
0 & \lambda \\
-\frac{1}{\lambda} & 0
\end{array}\right) .
$$

The group $\mathcal{G}$ consists of the identity component $\mathcal{G}_{0} \cong \mathbb{R}^{*}$ generated by $M_{0}(\lambda)$ and the connected components $\mathcal{G}_{i}$ consisting of matrices of the form $M_{i}(\lambda)$ for $i=1,2,3$. Denote $g_{i}=M_{i}(1)$ for $i=1,2,3$. Then $\mathcal{G}$ is generated by $M_{0}(\lambda)$ and the $g_{i}$.

The torsion subgroup of finite symmetries of $\operatorname{Aut}_{\text {lin }}(X)$ is $\mathbb{Z}_{2} \times H$, where $H$ denotes the subgroup of $\mathcal{G}$ that is generated by $g_{1}, g_{2}, g_{3}$. We easily compute that $H$ is isomorphic to $D_{4}$. Note that $\mathbb{Z}_{2} \times H$ is contained in $O_{3}(\mathbb{Z}) \subseteq G L_{3}(\mathbb{Z})$.

One can construct Helix similarly to Astrix in the appetizer section by symmetrization: First one deforms the circle $x^{2}+y^{2}=1$ in $\mathbb{A}^{2}$ by contracting the $y$-axis in $\mathbb{A}^{2}$ to a point via $(x, y) \rightarrow(x, x y)$. The resulting curve is the figure eight curve Lemniscate in $\mathbb{A}^{2}$ given by the equation $x^{4}+y^{2}=x^{2}$. Then consider the Cartesian product of the Lemniscate with a line. The so constructed surface $C$ lives in $\mathbb{A}^{3}$ and is still defined by the equation $x^{4}-x^{2}+y^{2}=0$. Then substitute $y$ by $y z$ in $C$ in order to obtain Helix. This last substitution corresponds to the restriction to $C$ of the blowup of $\mathbb{A}^{3}$ with center the $x$-axis. Notice that this axis is not contained in $C$ and that the blowup creates more complicated singularities.

This construction of Helix is very simple. It is, however, not clear how to invert it: Assume given Helix from scratch. We ask for the reconstruction of a smooth surface that projects birationally onto Helix as in the construction above. This will be a (nonembedded) resolution. Moreover, one can ask if the symmetries of Helix are preserved: Suppose $G \subseteq \operatorname{Aut}\left(\mathbb{A}^{3}\right)$ is a group acting on $X$. Is there a resolution $\pi: \widetilde{\mathbb{A}}^{3} \rightarrow \mathbb{A}^{3}$ such that this action can be lifted to the resolved $\widetilde{X} \subseteq \widetilde{\mathbb{A}}^{3}$ ? The answer to this question is 'yes' and was first given in arbitrary dimension by Villamayor [40. More precisely, if $G$ acts on $X \subseteq \mathbb{A}^{3}$ one can find a resolution of $X$ that is a composition of blowups in centers invariant under the action of $G$. Therefore,

\footnotetext{
${ }^{4}$ One knows for example that a polynomial map $\varphi: \mathbb{A}_{\mathbb{C}}^{n} \rightarrow \mathbb{A}_{\mathbb{C}}^{n}$ is an automorphism if and only if it is bijective; see [23, Lemma II.3.4]. Another interesting fact is that $\operatorname{Aut}\left(\mathbb{A}_{\mathbb{C}}^{2}\right)$ is the amalgamated free product of the subgroup of affine transformations $\operatorname{Aut}_{\text {aff }}\left(\mathbb{A}^{2}\right)=\left\{\varphi=\left(\varphi_{1}, \varphi_{2}\right) \in \operatorname{Aut}\left(\mathbb{A}^{2}\right): \varphi_{i}\right.$ affine linear $\}$ and the Jonquière subgroup $\mathfrak{J}_{2}=\left\{\varphi=\left(\varphi_{1}, \varphi_{2}\right) \in \operatorname{Aut}\left(\mathbb{A}^{2}\right): \varphi_{1} \in \mathbb{C}\left[x_{1}\right], \varphi_{2} \in\right.$ $\left.\mathbb{C}\left[x_{1}, x_{2}\right]\right\}$ over their intersection. This result is due to Jung [21 and Van der Kulk [39]. For higher dimensions the situation is much more complicated; see for example [24, 33,35 .
} 
the action can be lifted at each step of the resolution. Such a resolution is called equivariant.

To resolve Helix, there are four candidates for the first blowup. We discuss them separately.

(a) Blowup of origin.

(b) Blowup of line.

(c) Blowup of cross.

(d) Blowup of augmented cross.

(a) Blowup of the origin. This center is the only smooth subvariety of the singular locus which respects the symmetries of Helix. But it turns out to be too small to yield a clear improvement of the singularities. Let $X^{\prime}$ be the strict transform. The singularities of $X^{\prime}$ do not lie in the $x$-chart of the blowup. In the $y$-chart the strict transform is $X_{y}^{\prime}: x^{4} y^{2}+y^{2} z^{2}=x^{2}$; see Figure 21. The equation seems to be more complicated, and neither the singular locus has changed nor the order of the equation has dropped at any of the singular points of $X^{\prime}$. There is, however, a subtle improvement. The two singular axes of Helix have been separated. They can now be chosen simultaneously as the (smooth) center of the next blowup. Note that a blowup with center the $z$-axis of $X_{y}^{\prime}$ and the $y$-axis of $X_{z}^{\prime}$ will preserve the symmetries of $X$. In the strict transform $X^{\prime \prime}$, which is covered by 5 charts (one stemming from the $x$-chart of $X^{\prime}$, and two at a time from the $y$ - and $z$-chart), the singular locus consists of two one-dimensional components: the $y$-axis in $X_{y y}^{\prime \prime}$ and the $z$-axis in $X_{z z}^{\prime \prime}$. The intersection of these two lines is empty. Therefore we can blow them up simultaneously in order to obtain $X^{\prime \prime \prime}$. A computation shows that $X^{\prime \prime \prime}$ is already resolved. This resolution is not the most economic one but it is by construction equivariant.
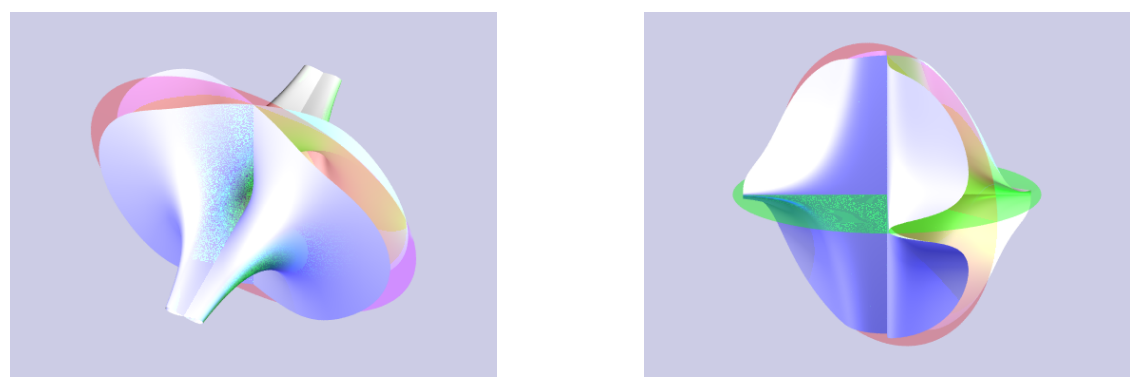

Figure 21. Blowup of Helix in the origin: $X^{\prime}$ after point blowup (left), and $X^{\prime \prime}$ after line blowup of $X^{\prime}$ (right).

(b) Blowup of line. The singular locus of Helix is the union of the $y$ - and the $z$-axis, the zeroset $V(x, y z)$. We have two equal choices for centers if we drop the requirement that the symmetries of $X$ should be preserved and just demand smooth centers: the $z$-axis and the $y$-axis. We illustrate the resolution by choosing the $z$ axis as the first center of the blowup (first blowing up the other axis would yield a symmetric blowup and can be obtained by permuting $y$ and $z$ ).

In the $x$-chart the strict transform $X_{x}^{\prime}$ is already smooth; see Figure 22. It intersects the exceptional plane $V(x)$ in two hyperbolas and has normal crossings with this plane. Thus Helix is resolved in this chart. 

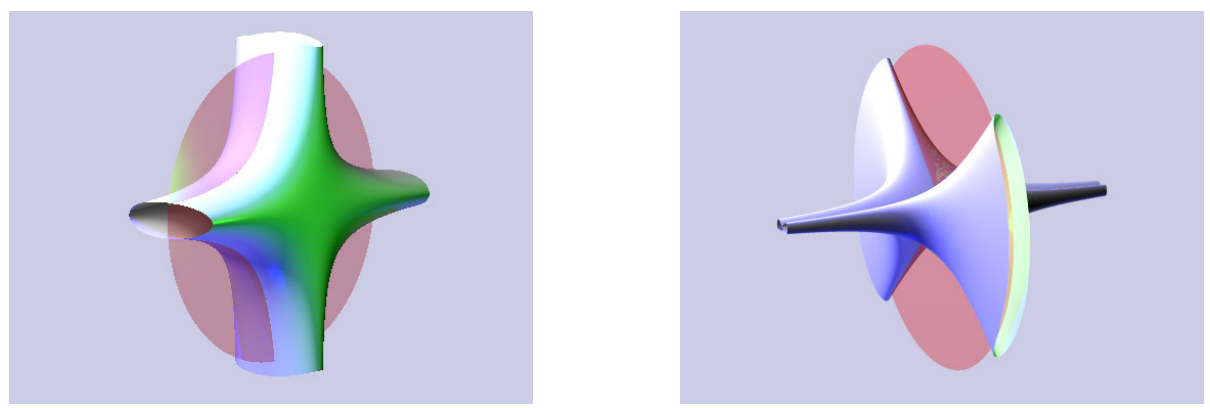

Figure 22. Blowup of Helix in the $z$-axis: $x$-chart (left) and $y$ chart (right), the exceptional component in red.

In the other chart the strict transform is singular, but the situation has clearly improved: the singular locus of $X_{y}^{\prime}$ is just the $y$-axis; see Figure 22 Thus we choose it as the center for the second blowup. In the $y x$-chart we get the same strict transform as in the $x$-chart of the preceding blowup (after the coordinate change $(x, y, z) \mapsto(z, y, x))$. The exceptional curves, i.e., the intersection curves of $X_{y x}^{\prime \prime}$ with the exceptional hyperplanes $V(x)$ and $V(y)$, are four distinct lines, which intersect pairwise transversally in two points on $X_{y x}^{\prime \prime}$; see Figure 23, left. A computation shows that $X^{\prime \prime}$ and the exceptional hyperplanes have normal crossings everywhere. In the $y z$-chart the total transform is defined by the equation $y^{2} z^{2}\left(x^{4} y^{2} z^{2}-x^{2}+1\right)=0$; see Figure 23, As in the $y x$-chart, the total transform has normal crossings everywhere. Helix is resolved after only two blowups. But the symmetry has been destroyed.
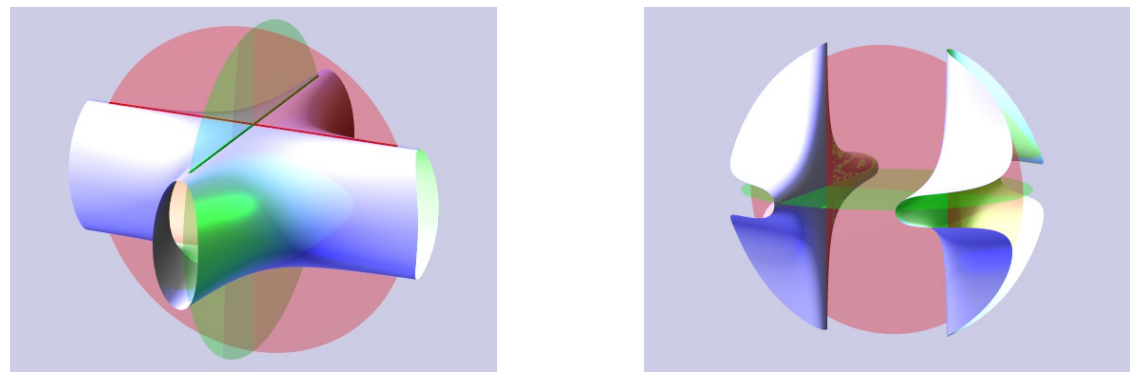

Figure 23. Blowup of Helix: second blowup of the axes, $y x$-chart (left), $y z$-chart (right).

(c) Blowup of the cross. How can we improve our resolutions by making as few blowups as possible? In (a) we needed four blowups, in (b) we cut down to two (losing the equivariance of the resolution), and now we try to resolve Helix in one step. One could think of resolving Helix with one blowup with center the whole singular locus of $X$, i.e., the union of the $y$ - and $z$-axis, with reduced ideal $(x, y z)$. We call this ideal the cross. A blowup with center the cross preserves the symmetries of Helix. But there is a substantial difference to the blowups from (a) and (b): the center itself is singular. A blowup $\pi: \widetilde{\mathbb{A}}^{3} \rightarrow \mathbb{A}^{3}$ is defined for any center that is a subvariety of $\mathbb{A}^{3}$, but for a singular center the blowup $\widetilde{\mathbb{A}}^{3}$ of the ambient space can also be singular. This is in particular the case for the cross. 
The $x$ - and the $y z$-chart cover $\widetilde{\mathbb{A}}^{3}$. In the latter chart the affine coordinate ring of the ambient space is $K\left[\frac{x}{y z}, y, z\right]$, and the strict transform of Helix is defined by the equation $x^{4} y^{2} z^{2}-x^{2}+1=0$ with exceptional components $V(y)$ and $V(z)$. The total transform coincides with $X_{y z}^{\prime \prime}$ of the preceding resolution; see Figure 23, left. The problems appear in the second chart. Here the affine coordinate ring is isomorphic to $K[x, y, z, w] /(x w-y z)$. Thus in this chart $\widetilde{\mathbb{A}}^{3}$ can be realized as a three-dimensional variety in $\mathbb{A}^{4}$ with an isolated singular point at the origin (a cone). The strict transform $X^{\prime}$ of Helix is given as $V\left(x w-y z, x^{2}-1+w^{2}\right) \subseteq \mathbb{A}^{4}$ in the $x$-chart. It does not touch the singularity of $\widetilde{\mathbb{A}}^{3}$ and even the exceptional curves on it are smooth. So Helix is resolved. One could fix the problem with the singular ambient space: in this example, the singularity of $\widetilde{\mathbb{A}}^{3}$ is resolved by blowing up its singular point. Since a blowup is an isomorphism outside the center, the transformation has no effect on the strict transform of Helix.

A blowup with center the cross resolves Helix. But in general the strict transform of a surface with singular locus the cross will meet the singular point in $\widetilde{\mathbb{A}}^{3}$ such that we will lose control of the singularities of the surface that we want to resolve. Nonetheless we can state

Problem 3. Let $X$ be a surface in $\mathbb{A}^{3}$ with $\operatorname{Sing}(X)=V(x, y z)$. Consider the blowup $\pi: \widetilde{\mathbb{A}}^{3} \rightarrow \mathbb{A}^{3}$ with center the cross $V(x, y z)$. Characterize all surfaces $X$ such that the strict transform $X^{\prime}$ does not meet the singular point of the ambient $\widetilde{\mathbb{A}}^{3}$.

(d) Blowup of the augmented cross. In the last approach we refine our method by equipping the ideal of our singular center with a nonreduced structure. By a clever choice of such a structure the ambient space remains smooth and, as an extra, Helix will be resolved in one step. We shall use the nonreduced ideal $(x, y z)(x, y)(x, z)=\left(x^{3}, x^{2} y, x^{2} z, x y z, y^{2} z\right)$ for the cross. This ideal will be called the augmented cross. The blowup in this center is the composition of the blowup of the cross in $\mathbb{A}^{3}$ followed by the blowup of the unique singular point of $\widetilde{\mathbb{A}}^{3}$. For the detailed description of this center and computations of the charts, see [14].

In each of the five charts the total transform of Helix has normal crossings. Thus Helix is resolved. In Figure 24 only three affine charts are depicted since the strict transform of Helix looks similar in the other two charts.
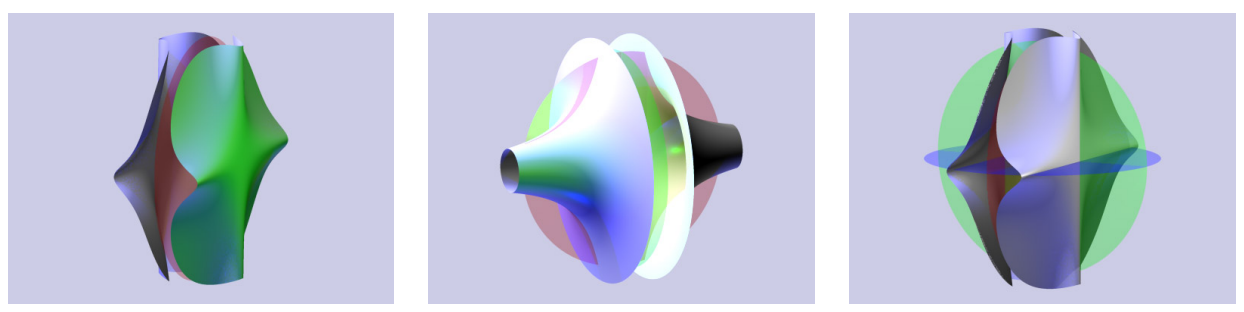

Figure 24. Blowup of Helix, center the augmented cross: the three relevant charts.

This resolution is the most economic one since one needs just one blowup and the ambient space remains smooth; moreover it respects all symmetries of Helix. However, it is not obvious how to generalize the resolution to higher dimensions or to a more complicated structure of the singular locus. 
Problem 4. Let $X$ be a singular surface in $\mathbb{A}^{3}$. Determine a (possibly singular and nonreduced) center to resolve $X$ with one blowup. Can you choose this center in a way that it respects the symmetries of $X$ ?

$$
\text { Simplicity: Sofa } x^{2}+y^{3}+z^{5}=0
$$

Let us relax and sit down on Sofa. It is perfectly suited for the dessert of our menu. Sofa is defined by the equation $f: x^{2}+y^{3}+z^{5}=0$ and looks nearly smooth except at the origin; see Figure 25. Its coordinate ring $K[x, y, z] /(f)$ is isomorphic to the invariant ring of the icosahedral symmetry group. Sofa has an isolated singularity at the origin, namely a simple singularity of type $E_{8}$ (the concept of simple singularities will be defined below). Although the singular locus is just a point, the (nonembedded) resolution of Sofa will be lengthy (in total, eight point blowups will be needed to achieve smooth strict transforms in all charts). After introducing simple singularities and their $A D E$-classification we will compute explicitly the resolution of Sofa by a sequence of point blowups. At the end of the section we will describe and construct the associated dual resolution graph.

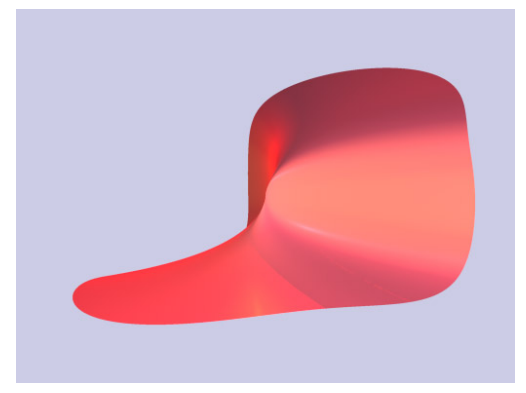

Figure 25. Sofa $x^{2}+y^{3}+z^{5}=0$.

We need some concepts from local analytic geometry. Let $\mathcal{O}_{n}=\mathbb{C}\left\{x_{1}, \ldots, x_{n}\right\}$ be the ring of convergent power series at 0 and consider the group $\mathcal{G}$ of local automorphisms of $\left(\mathbb{C}^{n}, 0\right)$. We write

$$
\mathcal{G} \times \mathcal{O}_{n} \rightarrow \mathcal{O}_{n},(\varphi, f) \mapsto \varphi \cdot f=f \circ \varphi^{-1}
$$

for the canonical group action. For an element $f \in \mathcal{O}_{n}$ the right equivalence class of $f$ is the orbit of $f$ under the above group action, denoted by $\mathcal{G} \cdot f=\{\varphi \cdot f: \varphi \in \mathcal{G}\}$. Let $\mathfrak{m}=\left\{f \in \mathcal{O}_{n}: f(0)=0\right\}$ be the maximal ideal of $\mathcal{O}_{n}$ and let $\pi_{k}: \mathcal{O}_{n} \rightarrow J_{k}=$ $\mathbb{C}\left\{x_{1}, \ldots, x_{n}\right\} / \mathfrak{m}^{k+1}$ be the projection map on the $k$-jet space $J_{k}$. Each $J_{k}$ is a finite-dimensional $\mathbb{C}$-vector space isomorphic to some $\mathbb{C}^{N}$ with the usual Euclidean topology on it. Take the coarsest topology on $\mathcal{O}_{n}$ such that all $\pi_{k}$ are continuous.

\footnotetext{
5 Some remarks on this problem: We know that there exists a sequence of blowups in smooth centers that resolve any algebraic variety in characteristic 0. By 13, Thm. 7.17], this sequence corresponds to one blowup in a possibly very complicated center. However, it is not clear how to find this center. It is in general singular and nonreduced, but even in concrete examples little is known. Similarly, it is quite hard to determine whether the blowup in a singular center leaves the ambient space smooth. For centers defined by monomial ideals the question is more tractable; see Rosenberg [34] and Faber 9]. Even if one succeeds in finding a nonreduced center such that the ambient space stays smooth, it is not obvious how to determine whether the singularities of the embedded subvariety improve.
} 
We say that an element $f$ is simple if there exists an open neighborhood $U$ of $f$ such that the number of orbits $\mathcal{G} \cdot f$ that intersect $U$ is finite. It is well known that simple germs $(V(f), 0)$ have an isolated singularity of order 2 at the origin, i.e., a double point; see [4, chapter 9]. Only a finite number of nonequivalent singularities appear in any of their deformations [4. Exercise 9.2.16].

Now we restrict our considerations to $\mathbb{C}^{3}$ and $f \in \mathbb{C}\{x, y, z\}$. Arnol'd has shown that every simple $f$ is right equivalent to one of the so-called $A D E$-singularities; see [1, 4, 6]. They come in two infinite families, $A_{k}: x^{2}+y^{2}+z^{k+1}$, for $k \geq 1$ and $D_{k}: x^{2}+y^{2} z+z^{k-1}$, for $n \geq 4$, together with the three exceptional singularities $E_{6}: x^{2}+y^{3}+z^{4}, E_{7}: x^{2}+y^{3}+y z^{3}, E_{8}: x^{2}+y^{3}+z^{5}$. A simple element $g$ is called adjacent to $f$ if $g$ is in the closure of the $\mathcal{G}$-orbit through $f$. This signifies that a small perturbation may deform $g$ into $f$. We thus get a partial order on the set of simple singularities according to their "complexity" [1, 25]:

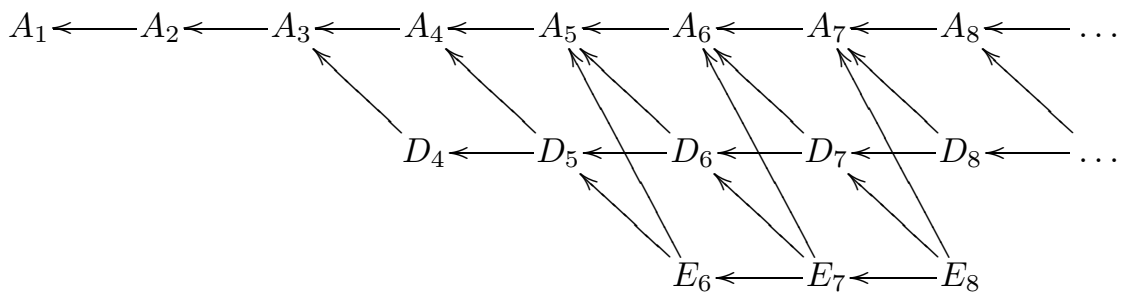

In a different vein, simple surface singularities can be characterized by their resolution: all simple singularities can be resolved by a sequence of point blowups. Such a singularity is called absolutely isolated. For example, an $A_{1}$-singularity is the ordinary cone $x^{2}+y^{2}=z^{2}$ and can be resolved by a single point blowup, whereas the $E_{8}$-singularity is quite complicated and requires, as mentioned above, eight point blowups. Kirby 22] has shown that an analytic hypersurface germ $(V(f), 0)$ is simple if and only if $f$ has an absolutely isolated double point. It is then interesting to see how the type of the singularity changes after each blowup: this change can be followed by subsequently deleting vertices from the associated dual resolution graph. As we will see later in this section, such a graph is uniquely determined for each $A D E$-singularity and codifies the combinatorics of its so-called minimal resolution. By computing the resolution of simple singularities case by case one can determine which vertices must be deleted.

There are several other characterizations of simple singularities, for example, as rational double points or quotient singularities. The articles of Greuel [12] and Durfee [6] provide an overview of these characterizations.

In this section we compute a nonembedded resolution, so that the last strict transform of $X$ does not need to have normal crossings with the exceptional divisor 6

Resolution of Sofa. Let us now describe how to resolve the $E_{8}$-singularity of the surface Sofa $S$ by a sequence of blowups. We will keep track of the blowups, the change of the type of the singularity and the exceptional curves and their intersections. The configuration of the exceptional curves on the strict transform is important for computing the Dynkin diagram associated to the singularity. This

\footnotetext{
${ }^{6}$ For surfaces, one could also obtain a resolution by repeatedly normalizing and then blowing up the singular points. This method is due to Zariski [44], based on the work by Jung [20]. The article of Lipman [29] gives a general overview of resolution techniques. Resolution of normal surface singularities is discussed in great detail in Laufer's book 26.
} 
diagram codifies the combinatorics of how the components of the exceptional divisor meet.

In the diagram below one can see how the type of the singularity changes under point blowups.

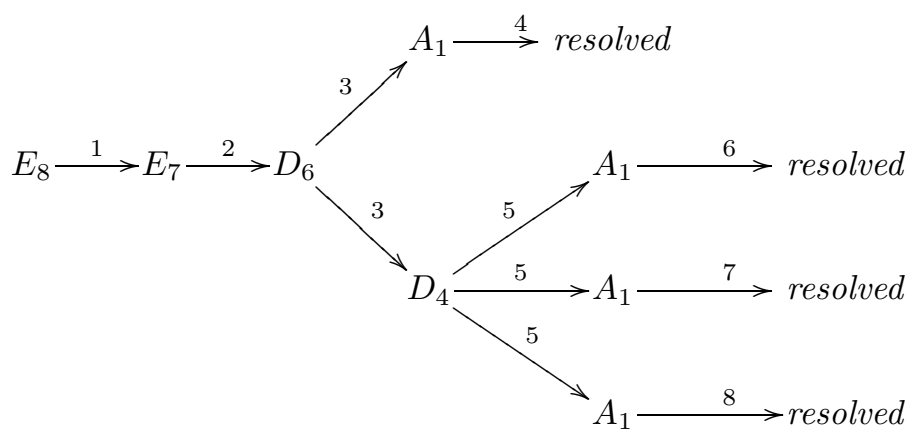

We see that the $E_{8}$-singularity is transformed into $E_{7}$ after the first blowup and into $D_{6}$ after the second blowup. Then the singularity splits up into two singularities: one $A_{1}$-singularity, which can be resolved by a single point blowup, and one $D_{4}$-singularity, which needs a total of four point blowups for its resolution. In the diagram below the strict transforms and centers are indicated:

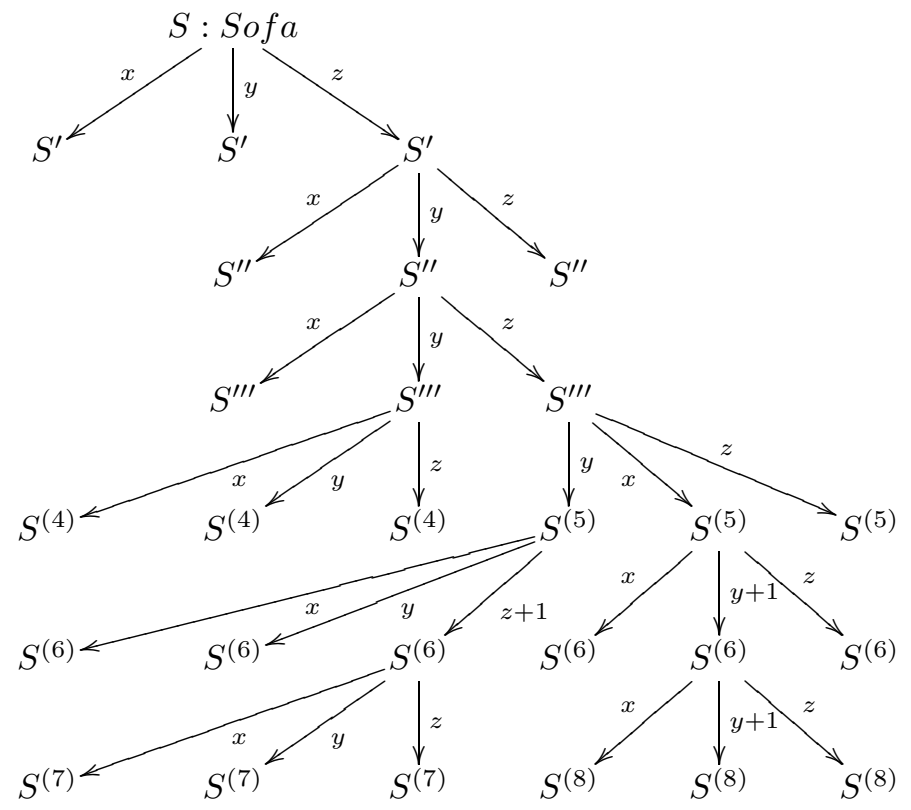

In the following we illustrate the resolution of Sofa (as in the diagram) more explicitly. We denote the components of the exceptional divisor $\mathbb{E}$ with $\mathbb{E}_{1}, \ldots, \mathbb{E}_{8}$, where $\mathbb{E}_{i}$ is the exceptional surface in the ambient space that is obtained by the $i$-th blowup. We denote the exceptional curves, i.e., the intersection of $\mathbb{E}_{i}$ and the strict transform, by $\mathbb{D}_{i}$. Sofa has an isolated singularity at the origin, which is the first center of the blowup. The resulting total transforms $S_{x}^{*}, S_{y}^{*}, S_{z}^{*}$ in the three charts are shown in Figure 26, Here the first exceptional surface $\mathbb{E}_{1}$ is shown in red. 

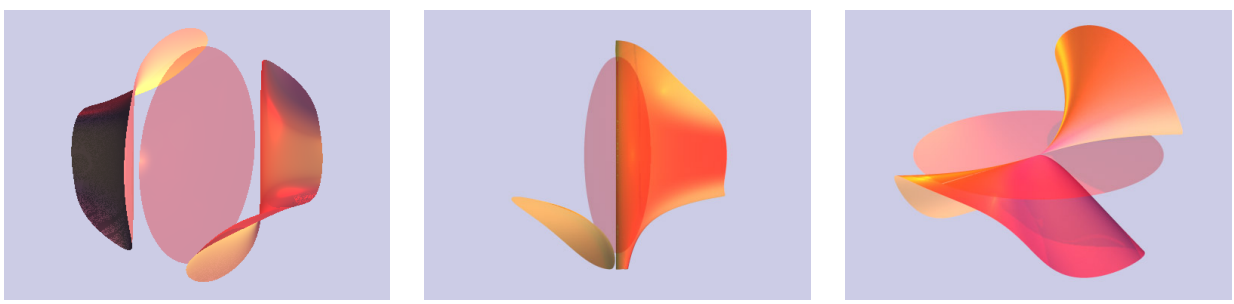

FigurE 26. The charts of the total transforms $S_{x}^{*}, S_{y}^{*}, S_{z}^{*}$ of Sofa.

We directly see that the strict transform in the $x$ - and $y$-chart is smooth. In the $y$-chart the total transform is defined by the equation $y^{2}\left(x^{2}+y+y^{3} z^{5}\right)=0$. It does not have normal crossings since the two components have a common tangent plane at 0 (the $x z$-plane). The exceptional $z$-axis $\mathbb{D}_{1}$ is smooth.

In the $z$-chart the strict transform $S_{z}^{\prime}$ is defined by the equation $x^{2}+y^{3} z+z^{3}=0$. This equation, which appears in Arnol'd's list of $A D E$-singularities [6, Table 1] as an $E_{7}$-singularity, is less complicated than $E_{8}$. We have only obtained a small success, so let us blow up the origin of $S_{z}^{\prime}$. Note that the origin is not contained in any other chart. Hence the other charts do not play a role in the further resolution and can be discarded.

The new total transforms $S_{z x}^{*}, S_{z y}^{*}, S_{z z}^{*}$ of Sofa are shown in Figure 27. The new exceptional component $\mathbb{E}_{2}$ is depicted in green.
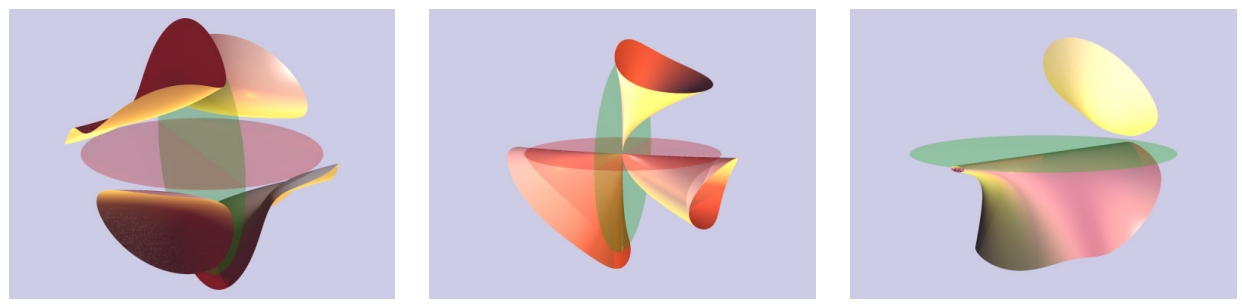

FiguRE 27. $S_{z x}^{*}, S_{z y}^{*}, S_{z z}^{*}$ : The three total transforms of $S_{z}^{*}$ under point blowup.

Now the strict transform $S^{\prime \prime}$ is smooth in two of the three new charts (the $z x$ and $z z$-chart). Moreover the exceptional curve $\mathbb{D}_{2}$ on $S_{z z}^{\prime \prime}$ is nonsingular and $S_{z x}^{\prime \prime}$ does not meet the exceptional divisor $\mathbb{E}_{1} \cup \mathbb{E}_{2}$.

In the $z y$-chart the strict transform $S_{z y}^{\prime \prime}$, which is defined by the equation $x^{2}+$ $y^{2} z+y z^{3}=0$, consists locally at the origin of three components. At first sight the singularity looks more complicated than the singularity of $S_{z}^{\prime}$. The defining polynomial of $S_{z y}^{\prime \prime}$ is not in the table of $A D E$-functions [6. Table 1] but it is right equivalent to a function that appears in the table. Applying the coordinate change $y \rightsquigarrow y-\frac{1}{2} z^{2}$, the equation $x^{2}+y^{2} z+y z^{3}=0$ is transformed into $x^{2}+y^{2} z-\frac{1}{4} z^{5}=0$. The singularity of this surface is of type $D_{6}$. The exceptional curve $\mathbb{D}_{2}$ on $S^{\prime \prime}$ is smooth and intersects the transform of the first exceptional curve $\mathbb{D}_{1}$ transversally in the origin of the $z y$-chart.

Since $S_{z x}^{\prime \prime}$ and $S_{z z}^{\prime \prime}$ are smooth we will proceed with the resolution only in the $z y$ chart. For the third blowup we choose the only reasonable center: the origin of the 
$z y$-chart. Figure 28 shows the three new chart expressions of the total transform with $\mathbb{E}_{3}$ painted in yellow.
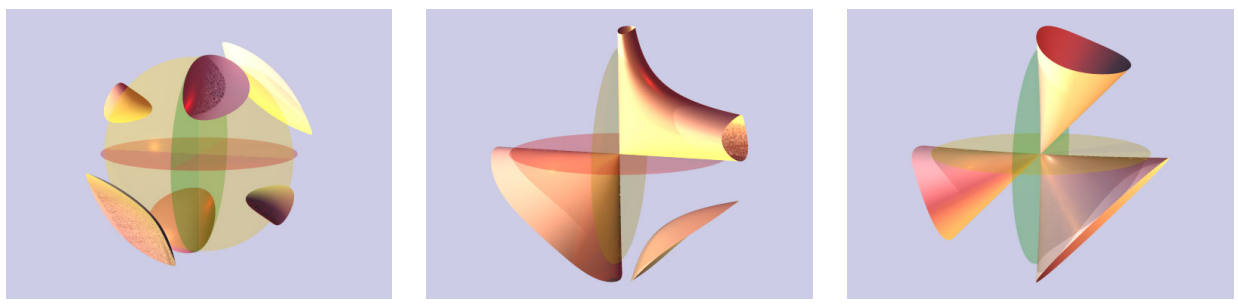

FiguRe 28. $S_{z y x}^{*}, S_{z y y}^{*}, S_{z y z}^{*}$ : The three chart expressions of the total transform of $S_{z y}^{*}$ under point blowup.

The strict transform $S^{\prime \prime \prime}$ is smooth in the $z y x$-chart; in the other two charts, it is still singular. There are two different singular points on $S^{\prime \prime \prime}$. The strict transform $S_{z y y}^{\prime \prime \prime}$ is defined by the equation $x^{2}+y z+y^{2} z^{3}=0$. It is clearly equivalent to $x^{2}+y z=0$, an $A_{1}$-singularity.

Before turning to the last chart of blowup number three, let us comment on the further resolution in the zyy-chart: on $S_{z y y}^{\prime \prime \prime}$ the exceptional curves $\mathbb{D}_{1}$ and $\mathbb{D}_{3}$ meet transversally in the singular point. The singular point can be resolved by one blowup. This blowup, being number 4, produces a new exceptional component $\mathbb{D}_{4}$, which intersects $\mathbb{D}_{1}$ transversally in the origin of the zyyy-chart of the strict transform and $\mathbb{D}_{3}$ transversally in the origin of the zyyx-chart. The exceptional curve $\mathbb{D}_{4}$ on $S^{(4)}$ does not meet $\mathbb{E}_{2}$.

Now we return to the chart of the third blowup that contains the worst singularity, namely the $z y z$-chart. Here the strict transform still consists of three "components". The origin of the strict transform $S_{z y z}^{\prime \prime \prime}$ defined by $x^{2}+y^{2} z+y z^{2}=0$ is of order 2 of type $D_{4}$. We have to blow up this surface a fifth time.

We blow up the only singular point of $S^{\prime \prime \prime}$, the origin of the $z y z$-chart. Now the singularity has clearly changed; see Figure 29. The new exceptional plane $\mathbb{E}_{5}$ is painted blue.
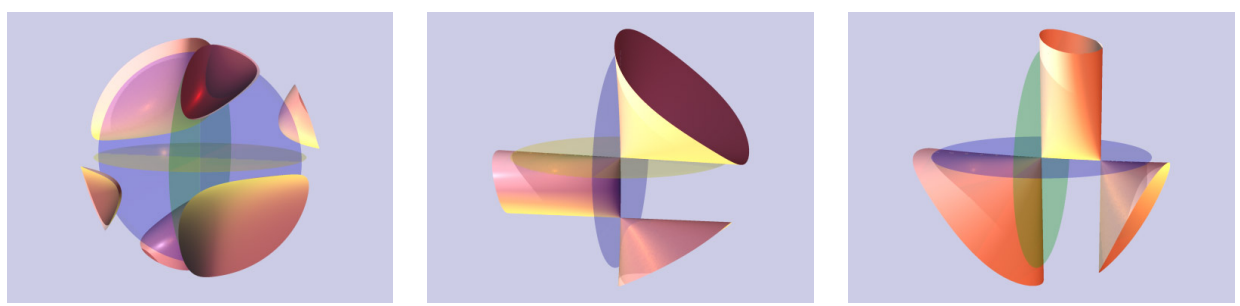

FIGURE 29. $S_{z y z x}^{*}, S_{z y z y}^{*}, S_{z y z z}^{*}$ : The three total transforms of $S_{z y z}^{*}$.

In the $z y z x$-chart the strict transform does not meet the exceptional components. But in the other two charts of $S^{(5)}$ we can see two remarkable things. First observation: the strict transforms $S_{z y z y}^{(5)}$ and $S_{z y z z}^{(5)}$ are isomorphic via the automorphism that interchanges $y$ and $z$. Second observation: the origin in both charts is still singular but we get another singular point in each chart, namely $V(x, y, z+1)$ 
in the $z y z y$-chart and $V(x, y+1, z)$ in the $z y z z$-chart. A computation shows that these two points globally correspond to the same point (use change of charts formula). Hence, on $S^{(5)}$ there are altogether three singular points: the origins of the two charts and an additional point. Thus the fifth blowup has finally separated the three "components" of $S_{z y z}^{\prime \prime \prime}$. All three singular points are of type $A_{1}$. Let us not forget the exceptional curves: in the $z y z y$-chart, $\mathbb{D}_{3}$ and $\mathbb{D}_{5}$ intersect transversally in one point and in the $z y z z$-chart, $\mathbb{D}_{5}$ and $\mathbb{D}_{2}$ intersect transversally in two different points.

For the sixth blowup we have two choices of the center in both charts: the origin and the new singular point. But each singularity will be resolved with one blowup since they are both of type $A_{1}$. We start with blowing up the new point $V(x, y, z+1)$ in the $z y z y$-chart. The other chart is similar. Two of the three resulting charts are displayed in Figure 30, this time the new exceptional component $\mathbb{E}_{6}$ is orange.

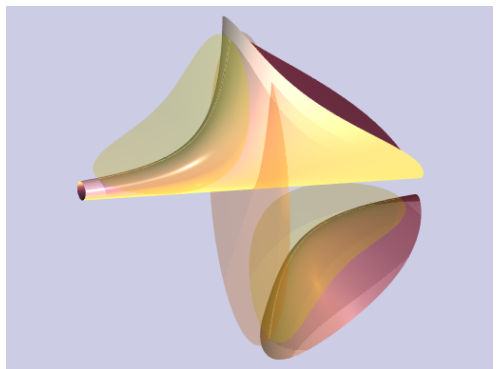

FIGURE 30. $S_{z y z y y}^{*}, S_{z y z y(z+1)}^{*}$ : The interesting transforms after the sixth blowup.

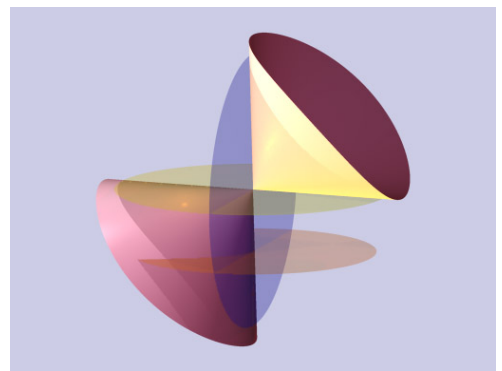

We do not provide a picture of the resolved $S_{z y z y x}^{*}$; it looks like all preceding resolved total transforms. The strict transform $X_{z y z y y}^{(6)}$ is smooth, and $\mathbb{D}_{6}$ can be seen as a smooth parabola in this chart; the other exceptional divisors do not intersect $S_{z y z y y}^{(6)}$. In the $z y z y(z+1)$-chart we can view our progress; there is only one singular point left, the origin. The equation of the strict transform $S_{z y z y(z)}^{(6)}$ is $x^{2}+y z=0$, a cone. Everything looks similar in the $z y z z(y+1)$-chart. Here the exceptional divisor $\mathbb{D}_{6}$ is a line and intersects $\mathbb{D}_{3}$ and $\mathbb{D}_{5}$ transversally in two different points. So we are ready for the final blowups, number seven and eight, of the last two singular points. In Figure 31 one of the three charts is displayed, namely $S_{z y z y(z+1) z}^{*}$, with $\mathbb{E}_{7}$ in violet.

All the other charts look quite similar (you may compute the equations yourself). It can be seen that the strict transform $S^{(8)}$ is smooth and in each chart the exceptional curves are smooth and meet pairwise transversally. Hence we are finished (except for the normal crossings of the strict transform and the exceptional divisor, which can be obtained by a line blowup). The intersections of the exceptional curves will be computed at the end of this section.

Resolution and Graphs. So far we have resolved Sofa via a sequence of blowups of points. We now turn to graphs, namely the Dynkin diagrams representing the resolution tree of $E_{8}$; see [20, 19, 26]. 


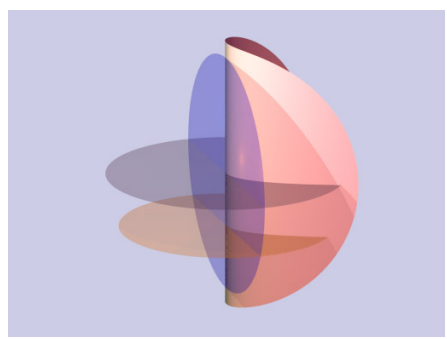

FIGURE 31. Finally smooth!

Consider a resolution $\pi: \widetilde{X} \rightarrow X$, where $X$ is a surface that has only isolated singularities and $\widetilde{X}$ is a manifold. Let $\mathbb{D}=\pi^{-1}(\operatorname{Sing}(X))$ denote the exceptional divisor. It consists of a finite union $\mathbb{D}_{1} \cup \ldots \cup \mathbb{D}_{s}$ of irreducible smooth curves. A resolution $\pi: \widetilde{X} \rightarrow X$ is called minimal if for any other resolution $\pi^{\prime}: \widetilde{X}_{1} \rightarrow X$ there exists a unique map $\rho: \widetilde{X}_{1} \rightarrow \tilde{X}$ such that $\pi^{\prime}=\pi \circ \rho$. Equivalently, a resolution is minimal if and only if it does not contain any rational irreducible exceptional curve $\mathbb{D}_{i}$ with self-intersection number $\mathbb{D}_{i} \cdot \mathbb{D}_{i}=-17$ For details see 26].

For every resolution $\pi: \tilde{X} \rightarrow X$ we can draw its dual resolution graph. It is a tree and encodes the combinatorics of the resolved surface $M$; one can read off the configuration of the exceptional curves from the graph. Let us assume that $\pi: \widetilde{X} \rightarrow X$ is a so-called good resolution. This means that the components of the exceptional divisor $\pi^{-1}(\operatorname{Sing}(X))$ intersect in a nice way (the exact definition can be found in the Appendix). Then a vertex $v$ corresponds to an irreducible component $\mathbb{D}_{v}$ of the exceptional divisor $\mathbb{D}$. Two vertices $v$ and $w$ are connected by $k$ edges if they intersect each other in $k$ distinct points. To each vertex $v$ we associate the self-intersection number of $\mathbb{D}_{v} \cdot \mathbb{D}_{v}$.

If $\pi$ is not a good resolution the construction of the resolution graph becomes more involved because we have to codify more combinatorial data. The details can be found in [26, 31. In these sources one can also find the resolution graph of $E_{8}$ :

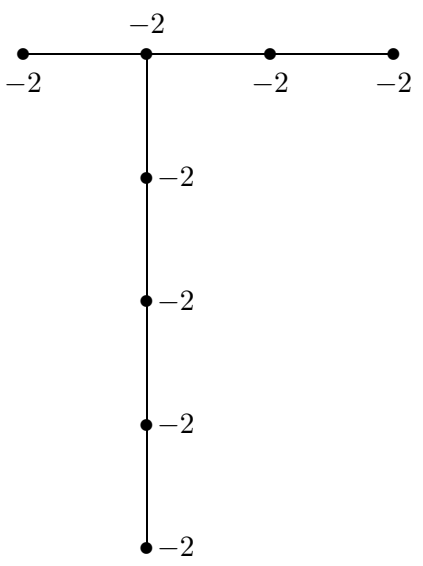

${ }^{7}$ This number is the first Chern class of the normal bundle of the embedding of $\mathbb{D}_{i}$ in $\widetilde{X}$. 
We compute the graph of our resolution of Sofa and see that it is equal to the graph of $E_{8}$. The computation of the self-intersection numbers can be found in [5]; the result is that $\mathbb{D}_{i} \cdot \mathbb{D}_{i}=-2$ for all $i$. This implies that our resolution is a minimal resolution 8

In the first part of this section we already remarked in which charts which exceptional divisors intersect. Now the results will be collected: the first charts in which exceptional curves intersect are (after the 4th blowup) the $z y y y$-chart and the zyyz-chart. Here $\mathbb{D}_{4}$ intersects $\mathbb{D}_{1}$, and $\mathbb{D}_{2}$ intersects $\mathbb{D}_{4}$ transversally in the origin of the respective chart. These are two different points on $S^{(4)}$. Hence the first part of the resolution tree looks as follows:

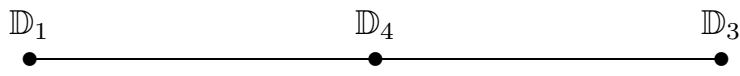

For the remaining charts we have to proceed to blowups number 7 and 8. Explicitly, the four relevant total transforms are: in the $z y z y(z+1) y$-chart the exceptional curves $\mathbb{D}_{7}=\{y=0\}$ and $\mathbb{D}_{3}=\{z=0\}$ intersect transversally on the strict transform $S_{z y z y(z+1) y}^{(8)}$ given as $V\left(x^{2}+z\right)$. Similarly $\mathbb{D}_{8}=\{z=0\}$ and $\mathbb{D}_{2}=\{y=0\}$ intersect the strict transform $S_{z y z z(y+1) z}^{(8)}$ given by $V\left(x^{2}+y\right)$ transversally. On the strict transform $S_{z y z z(z+1) z}^{(8)}$ the exceptional curve $\mathbb{D}_{5}=\{y=0\}$ intersects $\mathbb{D}_{7}=\{z=0\}$ in the origin and it intersects $\mathbb{D}_{6}=\{z+1=0\}$ in the point $(0,0,-1)$. In the $z y z z(y+1) y$-chart, $\mathbb{D}_{5}=\{z=0\}$ intersects $\mathbb{D}_{8}=\{y=0\}$ in the origin and it intersects $\mathbb{D}_{6}=\{z+1=0\}$ in the point $(0,-1,0)$. A computation shows that $(0,0,-1)$ and $(0,-1,0)$ correspond globally to the same point, so $\mathbb{D}_{5}$ intersects $\mathbb{D}_{6}$ transversally in one point. The resolution tree has the required form:

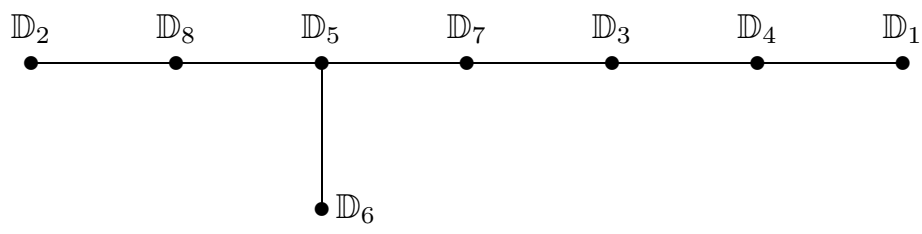

Singularity: Daisy $\left(x^{2}-y^{3}\right)^{2}=\left(z^{2}-y^{2}\right)^{3}$

We are pleased to offer a digestif. Suppose that now, instead of eliminating singularities, we want to produce singularities. More precisely, we intend to construct a surface with certain prescribed types of singularities. Until now, the surfaces had points or lines as the singular locus. Can we find surfaces whose singular locus is itself a singular curve? Moreover, can we prescribe the geometry of the surface along this curve, for instance, its generic transversal section with a plane perpendicular to the singular locus?

\footnotetext{
8 One can show that a minimal resolution of the singularities of a normal surface by a sequence of point blowups (and possibly normalizing) can only be achieved if the surface has rational singularities; see [28].
} 
There seems to be no systematic method to answer these questions. We shall give a partial solution by using the inverse process to blowups, so-called blowdowns. These correspond to contractions of subvarieties of dimension 2 of the ambient $\mathbb{A}^{3}$ and allow us to produce a certain range of singularities. We exhibit these constructions in a concrete example. However, there is still a lot of trial and error involved.

First have a look at the following surface, called Daisy (see Figure 32):

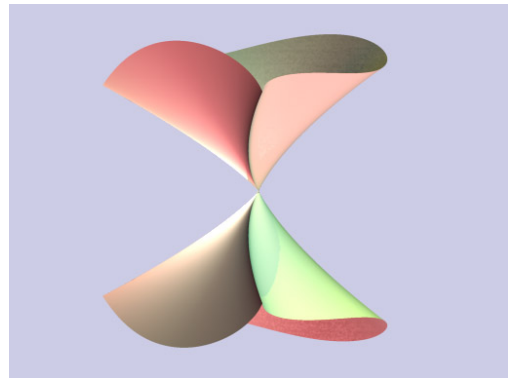

Figure 32. Daisy $\left(x^{2}-y^{3}\right)^{2}=\left(z^{2}-y^{2}\right)^{3}$.

This surface looks like a double cone which is folded along four edges. These edges form its singular locus: $\operatorname{Sing}(D)$ consists of two plane cusps meeting "transversally" at their common singular point. The cusps are the zerosets of the two ideals $\left(x^{2}-y^{3}, y \pm z\right)$.

Now assume that by mistake we have lost or forgotten the equation for Daisy. How can we recover it from the geometric appearance of the surface? We shall use a procedure similar to the construction of the surface Eighty: Start with the cylinder over the ellipse $x^{2}-2 x y+2 y^{2}-1=0$. Fold it along two parallel lines by contracting the two tangential planes $y=-1$ and $y=1$ in $\mathbb{A}^{3}$ to lines via the rational contraction map $\varphi_{1}: \mathbb{A}^{3} \rightarrow \mathbb{A}^{3},(x, y, z) \mapsto\left((x-y) /\left(y^{2}-1\right), y, z\right)$. The singular locus of the resulting "cylinder" (this is now a cylinder over the singular plane curve with equation $x^{2}+3 y^{2}-2 x y^{3}-3 y^{4}+2 y^{6}=1$ ) has two line components; see Figure 33 .
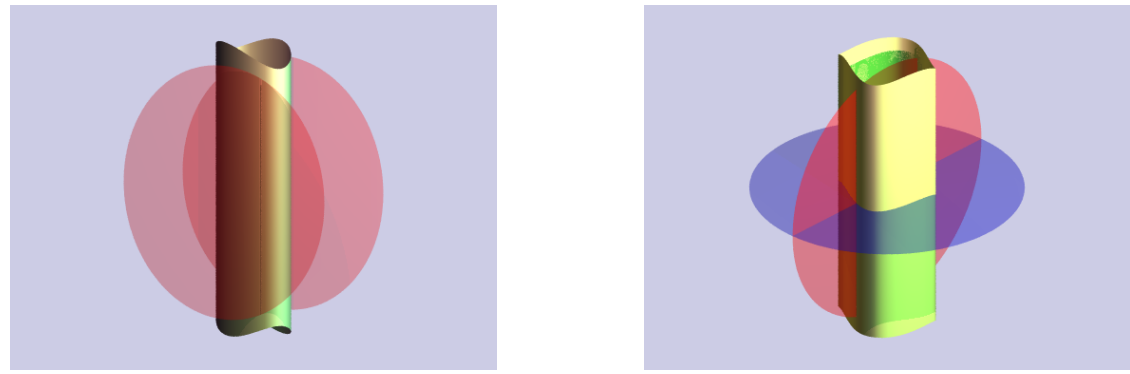

Figure 33. Construction of Daisy: Two tangent planes (left), and the contracted surface together with the $x y$ - and the $y z$-plane (right). 
In the next step we produce a new singular line on our surface by contracting the $x y$-plane in $\mathbb{A}^{3}$ (blue in Figure 33 ) to a line, the $y$-axis, via the contraction $\operatorname{map} \varphi_{2}: \mathbb{A}^{3} \rightarrow \mathbb{A}^{3},(x, y, z) \mapsto(x / z, y, z)$. Now the singular locus of this surface, defined by the equation $x^{2}-2 x y^{3} z-z^{2}+3 y^{2} z^{2}-3 y^{4} z^{2}+2 y^{6} z^{2}=0$, consists of three lines that intersect pairwise cleanly. Then we transform the "old" singular lines into parabolas by contracting the $x y$-plane in $\mathbb{A}^{3}$ to the $y$-axis via $\varphi_{3}: \mathbb{A}^{3} \rightarrow$ $\mathbb{A}^{3},(x, y, z) \mapsto(x, y, z / x)$. In the last step, the $x y$-plane is contracted to the origin via $\varphi_{4}: \mathbb{A}^{3} \rightarrow \mathbb{A}^{3},(x, y, z) \mapsto(x / z, y / z, z)$. The result is the surface Daisy.

The construction of a surface $X$ with a given plane curve $Y$ as its singular locus can be realized through the following recipe. Let $Y$ be given by the equation $f(x, y)=0$ and denote $I_{Y}=(f, z) \subseteq K[x, y, z]$. Choose a surface $S: g(x, y)-z^{k}=$ 0 , for $k \geq 0$, such that the singular locus of the plane curve $g(x, y)=0$ in $\mathbb{A}^{2}$ is either contained in $Y$ or does not meet $Y$. Then we define $X: g f^{k}-z^{k}=0$. The surface $S$ then corresponds to the $f$-chart expression of the blowup of $X$ when we use $I_{Y}$ as center.

The next interesting case is to search a surface with a given space curve $Y$ as its singular locus. The blowup of $\mathbb{A}^{3}$ with center $Y$ will in general be singular in each chart (each chart corresponds to a defining equation for $Y$ ). In particular, the chart expressions of the associated blow-up maps will not be maps from $\mathbb{A}^{3} \rightarrow \mathbb{A}^{3}$. Consider the following example:

Example 3. We construct a surface $X$ whose singular locus is the singular space curve $Y$ parametrized by $\left(t^{3}, t^{4}, t^{5}\right)$, in $\mathbb{A}^{3}$. This curve is ideal-theoretically not a complete intersection since its defining ideal $I=\left(x^{3}-y z, y^{2}-x z, z^{2}-x^{2} y\right)$ is minimally generated by three polynomials. Consider the blowup of $\mathbb{A}^{3}$ with center $I$. The resulting ambient space $\widetilde{\mathbb{A}}^{3}$ is singular in all three charts. Look at the $\left(y^{2}-x z\right)$-chart. Its coordinate ring is $K\left[x, y, z, \frac{x^{3}-y z}{y^{2}-x z}, \frac{z^{2}-x^{2} z}{y^{2}-x z}\right]$. We take as Ansatz the surface $X^{\prime}: x^{2}+y-z^{2}=0$ in $\mathbb{A}^{3}$ and substitute $\phi:(x, y, z) \mapsto$ $\left(\frac{x^{3}-y z}{y^{2}-x z}, y^{2}-x z, \frac{z^{2}-x^{2} y}{y^{2}-x z}\right)$ to find a surface $X$ in $\mathbb{A}^{3}$, which is defined by the equation $\left(x^{3}-y z\right)^{2}+\left(y^{2}-x z\right)^{3}-\left(z^{2}-x^{2} y\right)^{2}=0$. Indeed, $X$ has the singular space curve $Y$ as singular locus (see Figure 34, left)!

Example 4 (this is due to Robert Bryant). One can construct a surface $X$ with the same twisted space curve $Y$ as singular locus as in example 3 by using a different method. The curve $Y$ is defined in $\mathbb{A}^{3}$ by the ideal $I=\left(x^{3}-y z, y^{2}-x z, z^{2}-x^{2} y\right)$. Putting the weights 3,4 and 5 on $x, y$ and $z$ this ideal is weighted homogeneous. For $X=V(f)$ having singular locus $Y$ we will therefore require that $\sqrt{J(f)}=I$, where $J(f)$ denotes the Jacobian ideal of $f$ generated by the partial derivatives. It is now plausible to look for a weighted homogeneous $f$. By the Euler relation, any weighted homogeneous polynomial $f$ is contained in $J(f)$. Since we want $f \in \sqrt{J(f)}$ we take $f$ as a $K[x, y, z]$-linear combination of the generators of $\sqrt{J(f)}$ as Ansatz, i.e., $f=a \cdot\left(x^{3}-y z\right)+b \cdot\left(y^{2}-x z\right)+c \cdot\left(z^{2}-x^{2} y\right)$, with $a, b, c \in K[x, y, z]$. These polynomials should again be weighted homogeneous and have a weighted degree so that $f$ is also weighted homogeneous. Increasing successively the degrees of $a, b$ and $c$ the first feasible choice turns out to be $a=x^{2}, b=x y$ and $c=z$. This results in $f=x^{5}+x y^{3}+z^{3}-3 x^{2} y z$. The surface $X=V(f)$ is called Clip (see Figure [34, right). Unfortunately, we do not see the singular curve $Y$ here, since outside the origin the surface is a $C^{1}$-manifold. So even though the construction 
was neat, we will have to keep an eye on obtaining the desired local geometry of the surface at its singular points.
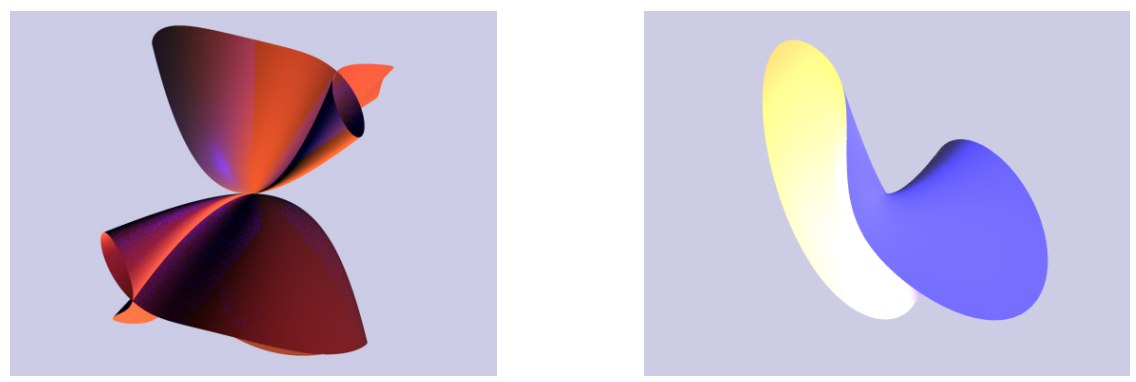

Figure 34. Twisted space curve as singular locus: Construction by contraction (left), and by weighted homogeneous Ansatz (right).

Problem 5. Let $Y$ be an irreducible curve in $\mathbb{A}^{3}$. Give a systematic construction of a surface $X$ for which $\operatorname{Sing}(X)=Y$ holds. Is it furthermore possible to prescribe the geometry type of a transversal section along the singular locus?

After this short excursion to the construction of singular surfaces we will resolve Daisy. Here is the diagram of blowups and charts expressions required for the resolution.

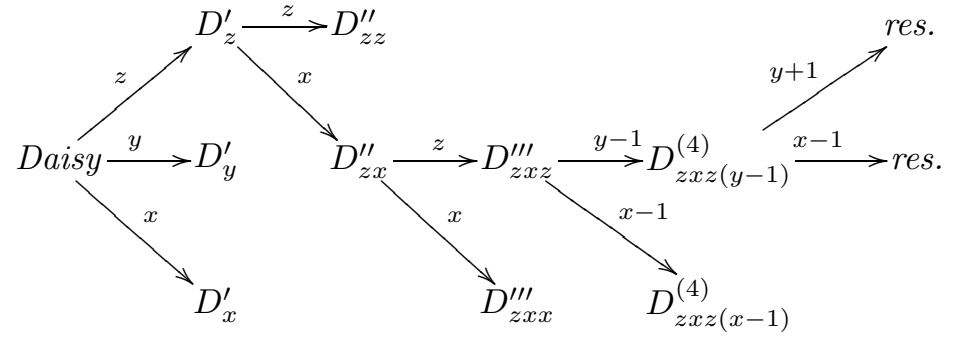

The leaves with label " $D_{a b c}$ " do not signify that Daisy is resolved in the corresponding chart. We just do not pursue the resolution in these charts because the singularities are not so interesting there. We will rather focus on the most important charts in the resolution of Daisy. A quick overview of our partial resolution: The singular locus of Daisy consists of two singular curves. It is not clear how to control the blowup of $\mathbb{A}^{3}$ and of Daisy if we take these curves as center. There would appear new singularities. Instead, we will follow the traditional approach; we will only use regular centers for blowups.

The first blowup will be a point blowup with center the origin, since it is the most singular point of our surface. Our preliminary goal will be to separate the two components of the singular locus of Daisy and to make them then smooth. The blowup has three charts; see Figure 35.

In the $x$-chart (left) the singular locus of the strict transform consists of two smooth curves, which do not intersect. In the $y$ - and $z$-chart we get similar pictures; the strict transforms have singular loci that consist of the union of a line and two smooth plane parabolas. In the $y$-chart the pairwise intersections of these three 

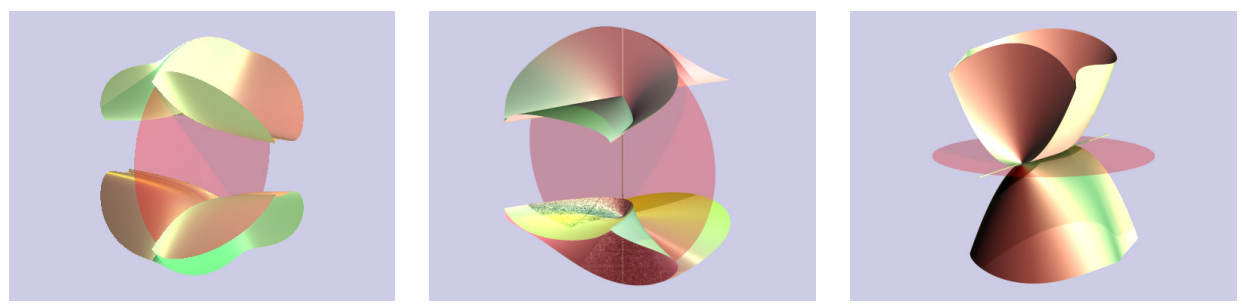

Figure $35 . D_{x}^{*}, D_{y}^{*}, D_{z}^{*}$ : The three total transforms of Daisy under the blowup of the origin (exceptional plane in red).

curves are empty, and in the $z$-chart the intersection of the two parabolas, which are in $\operatorname{Sing}(D)$, is empty and the line intersects each parabola in one point. Therefore the strict transform of Daisy is not smooth after the first blowup. The singularities are most involved in the $z$-chart. In the visualization of $D_{z}^{\prime}$ in $\mathbb{A}_{\mathbb{R}}^{3}$ one can see very well how Daisy is altered after the blowup of the origin. We therefore proceed in this chart.

In the $z$-chart we have three possible choices for the next center. We recall that we just blow up points or smooth curves inside the singular locus, which consists of the $y$-axis and the two parabolas. We choose the $y$-axis because then no symmetries of the strict transform will be destroyed. We get two new charts:

In the $z z$-chart the singular locus is the union of two hyperbolas, whose intersection is empty; see Figure 36. In the $z x$-chart the progress is small. The singular locus of the strict transform $D_{z x}^{\prime \prime}$ still consists of three components, namely the $y$-axis again and the two parabolas, which have been transformed into two lines. We proceed with blowing up in the $z x$-chart.
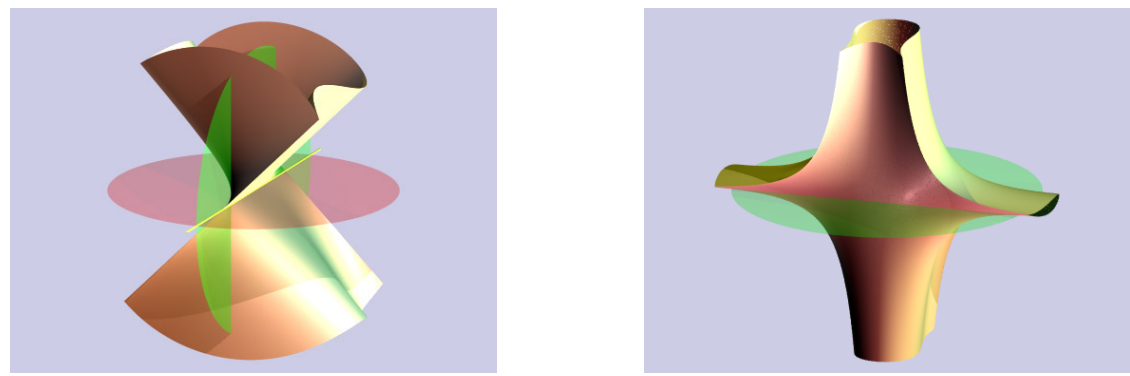

Figure 36. $D_{z x}^{*}, D_{z z}^{*}$ : The second transforms of Daisy in the $z x$ chart (left) and $z z$-chart (right), with the new exceptional plane in green.

There are three natural choices for the center of the third blowup: the two lines or the $y$-axis. We choose the $y$-axis as center; see Figure 37 There appears a significant improvement of the singularities of $D^{\prime \prime \prime}$. The singular locus consists now of two disjoint lines. The strict transform $D_{z x z}^{\prime \prime \prime}$ is a Cartesian product of a plane curve with two cusps and the $z$-axis.

We will proceed in the $z x z$-chart, where we choose as center one of the two singular lines lying in this chart, e.g. $x=y=1$. In the resulting $z x z(x-1)$-chart 

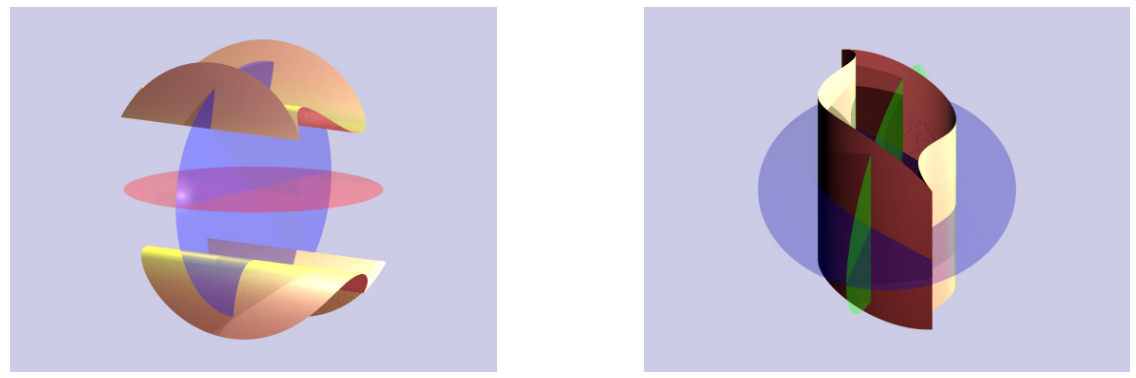

FiguRE 37. $D_{z x x}^{*}, D_{z x z}^{*}$ : The third transforms of Daisy in the $z x x$ chart (left) and $z x z$-chart (right), the new exceptional plane in blue.

the strict transform looks as if the cylinder had been torn apart. The singular locus of $D_{z x z(x-1)}^{(4)}$ is just one line, parallel to the $z$-axis. The exceptional components are the three planes $x=0, x=1$ and $z=0$. Clearly, they intersect transversally. The strict transform in the other chart of this fourth blowup behaves similarly; see Figure 38
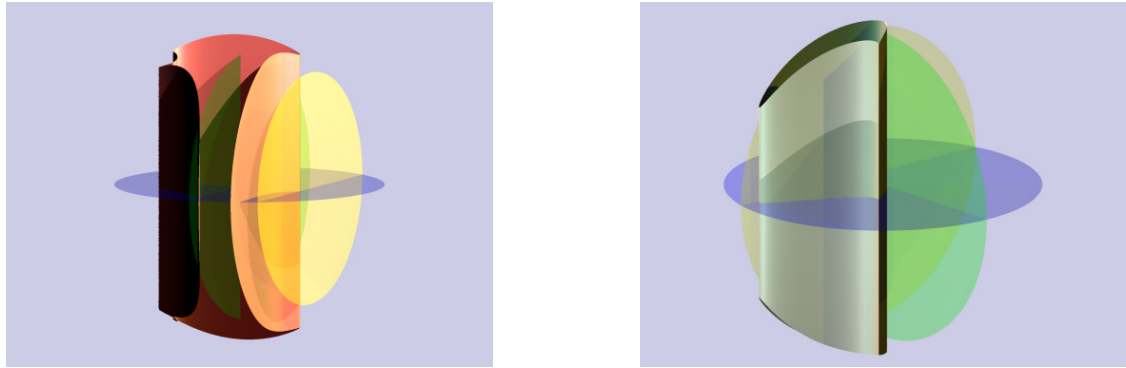

FiguRE 38. $D_{z x z(x-1)}^{*}, D_{z x z(y-1)}^{*}$ : The fourth total transforms of Daisy in the two charts of the fourth blowup with the new exceptional divisor in yellow.

As the two charts have similar singular loci, we perform blowup number 5 in that chart in which the equations are the easiest: the $z x z(x-1)$-chart. Then the "canonical" center is the singular line $x-1=y+1=0$. The blowup produces two new charts; see Figure 39

In the $z x z(y-1)(x-1)$-chart the strict transform is smooth. The bad news is that the intersection with the exceptional components is not smooth. We would have to perform more blowups to achieve mikado or normal crossings. In the $z x z(y-1)(y+$ 1)-chart the surface is a Cartesian product of the ellipse $2 y^{2}-2 x y+x^{2}=1$ with the $z$-axis and therefore smooth. But the exceptional components $y=1$ and $y=-1$ are tangent since the intersection consists of the two "double" lines defined by the ideals $\left(y-1,(x-1)^{2}\right)$ and $\left(y-1,(x+1)^{2}\right)$. To achieve normal crossings one first has to make the intersections with the exceptional components transversal; resp., one has to separate the components. This is a combinatorial problem that we do not pursue further here. 

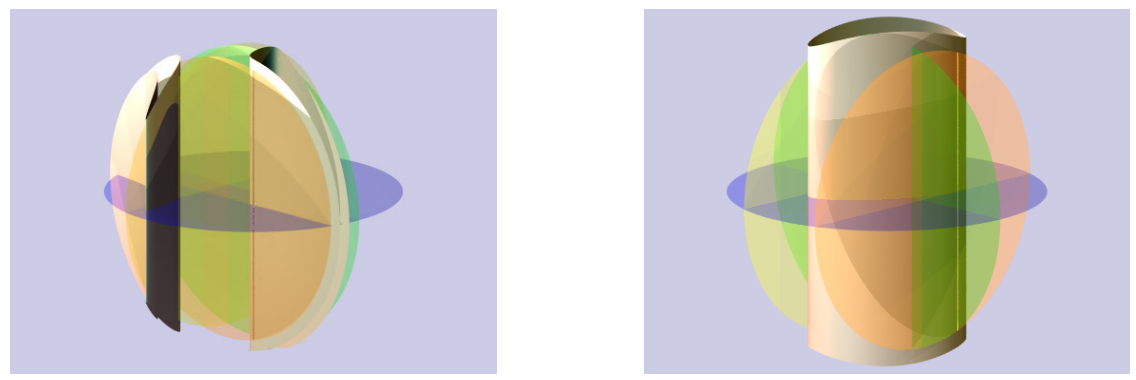

FIGURE 39. $D_{z x z(y-1)(x-1)}^{*}, D_{z x z(y-1)(y+1)}^{*}$ : The total transforms of Daisy in the two charts of the fifth blowup with the exceptional plane in orange.

\section{APPENDIX: BASIC CONCEPTS}

Throughout this text, $K$ denotes a fixed perfect field and $\mathbb{A}_{K}^{n}=\mathbb{A}^{n}$ denotes the affine $n$-space over $K$. The Zariski topology on $\mathbb{A}^{n}$ is defined by taking algebraic subsets of $\mathbb{A}^{n}$ as closed sets, i.e., sets of the form $V(S)=\left\{p \in \mathbb{A}^{n}: f(p)=0\right.$ for all $f \in S\}$, where $S$ is any set of polynomials in $K\left[x_{1}, \ldots, x_{n}\right]$. Let $X=V\left(f_{1}, \ldots, f_{k}\right)$, where $f_{i} \in K\left[x_{1}, \ldots, x_{n}\right]$, be an algebraic variety in $\mathbb{A}^{n}$. We assume that the ideal of $K\left[x_{1}, \ldots, x_{n}\right]$ generated by the $f_{i}$ is a radical ideal. A point $p \in X$ is then called smooth or regular if the rank of the matrix $\partial f_{i}\left(x_{j}\right)$ at $p$ equals $n-r$, where $r$ denotes the (Krull-)dimension of $X$ at $p$ (which is the topological dimension of $X$ with respect to the Zariski topology). The remaining points of $X$ are called singular points. They form a closed subset of $X$, the singular locus of $X$, denoted by $\operatorname{Sing}(X)$.

When speaking of the tangent space $T_{p}(X)$ in our algebraic context we mean the Zariski tangent space. In $\mathbb{A}^{n}$ it is defined by the system of linear equations

$$
\sum_{i=1}^{n} \frac{\partial f_{j}}{\partial x_{i}}(p)\left(x_{i}-p_{i}\right)=0, \text { for } j=1, \ldots, k
$$

A local ring $R$ of (Krull)-dimension $n$ with maximal ideal $\mathfrak{m}$ is called regular if $\mathfrak{m}$ can be generated by $n$ elements. Such a system of generators is minimal and then called a regular system of parameters for $R$. By a system of coordinates $x=\left(x_{1}, \ldots, x_{n}\right)$ around $p \in \mathbb{A}^{n}$ we mean that the $x_{i}$ form a regular system of parameters of the regular local ring $\mathcal{O}_{\mathbb{A}^{n}, p}=\left\{f / g: f, g \in K\left[x_{1}, \ldots, x_{n}\right]\right.$ and $g(p) \neq$ $0\}$.

Let $X_{1}, \ldots, X_{k}$ in $\mathbb{A}^{n}$ be a collection of closed algebraic varieties. We say that $X_{1}, \ldots, X_{k}$ have normal crossings at a point $p \in \mathbb{A}^{n}$ if there exists a system of coordinates locally around $p$, such that each $X_{i}$ is either defined as the zeroset of some of the coordinates or it is empty. We say that $X_{1}, \ldots, X_{k}$ have normal crossings in $\mathbb{A}^{n}$ if they have normal crossings at any point of $\mathbb{A}^{n}$.

Let $\mathbb{E}$ be the exceptional divisor of a blowup of a variety $X \subseteq \mathbb{A}^{n}$, and let $Y$ be a variety. We say that $Y$ is transversal to the exceptional locus $\mathbb{E}$ if $Y$ and the components of $\mathbb{E}$ have normal crossings. 
Stratification. Let $X$ be a variety over $\mathbb{R}$ or $\mathbb{C}$ of dimension $d$. A filtration of $X$ is a sequence of subvarieties

$$
X=Z_{d} \supseteq Z_{d-1} \supseteq \cdots \supseteq Z_{1} \supseteq Z_{0},
$$

such that each difference $Z_{i}-Z_{i-1}$ is an $i$-dimensional smooth open subvariety of $Z_{i}$, or is empty. Each connected component $X_{\alpha}$ of $Z_{i}-Z_{i-1}$ is called a stratum. Hence $X$ is a disjoint union of smooth strata; the collection of strata $\left\{X_{\alpha}\right\}_{\alpha \in A}$ is called a stratification of $X$. It satisfies the frontier condition if for all $\alpha, \beta$ such that $X_{\alpha} \cap \bar{X}_{\beta} \neq \emptyset$, one has $X_{\alpha} \subseteq \bar{X}_{\beta}$. Since the strata are disjoint this means that $X_{\alpha}=X_{\beta}$ or that $X_{\alpha} \subseteq \overline{X_{\beta}} \backslash X_{\beta}$. In the latter case we call $\left(X_{\alpha}, X_{\beta}\right)$ adjacent.

Now let $X$ be embedded in a smooth ambient variety $M$. Working locally we assume that $M$ is the affine space $\mathbb{A}^{n}$ over $\mathbb{R}$ or $\mathbb{C}$. An adjacent pair $\left(X_{\alpha}, X_{\beta}\right)$ satisfies Whitney's condition (a) at $y \in X_{\beta}$, if for all sequences $\left\{x_{i}\right\} \in X_{\alpha}$ convergent to $y \in X_{\beta}$ such that the sequence of tangent spaces $\left\{T_{x_{i}}\left(X_{\alpha}\right)\right\}$ converges (in the Grassmannian of $\operatorname{dim} X_{\alpha}$-subspaces of $\mathbb{A}^{n}$, or, more accurately, of the tangent bundle $T \mathbb{A}^{n}$ of $\left.\mathbb{A}^{n}\right)$ to a space $T$, we have $T_{y}\left(X_{\beta}\right) \subseteq T$. The pair $\left(X_{\alpha}, X_{\beta}\right)$ is said to satisfy Whitney's condition (b) along $X_{\beta}$ at $y \in X_{\beta}$ if given a sequence of points $\left\{x_{i}\right\}$ in $X_{\alpha}$ converging to $y$ and a sequence of points $\left\{y_{i}\right\}$ in $X_{\beta}$ also converging to $y$, and such that again $\left\{T_{x_{i}}\left(X_{\alpha}\right)\right\}$ converges to a space $T$, and also the secants $\overline{x_{i} y_{i}}$ in $\mathbb{A}^{n}$ converge to a line $l$, we have $l \subseteq T$. It can be shown easily that condition (b) implies (a); see [42].

A stratification $X=\bigcup_{\alpha \in A} X_{\alpha}$ satisfying the frontier condition and Whitney's conditions (a) and (b) at the points of the smaller stratum of any adjacent pair $\left(X_{\alpha}, X_{\beta}\right)$ is called a Whitney stratification. For different types of stratifications we refer to 38 .

Blowup. We start with the most intuitive definition of a blowup, the blowup of a point in a plane. We may assume that we blow up $\mathbb{A}^{2}$ in the origin. The idea is to lift all lines through the origin to $\mathbb{A}^{2} \times \mathbb{P}^{1}$ by associating to each point $(x, y)$ the slope $z$ of the line through 0 and the point $(x, y)$. This means to consider the map

$$
\sigma: \mathbb{A}^{2} \backslash\{0\} \rightarrow \mathbb{A}^{2} \times \mathbb{P}^{1},(x, y) \mapsto(x, y,(x: y)) .
$$

By taking the third coordinate in projective space, the slopes $\frac{y}{x}$ and $\frac{x}{y}$ are treated on equal footing. So we have to consider two affine charts of the blowup. Since the image of the origin is still missing, we define the blowup of $\mathbb{A}^{2}$ with center the origin, denoted $\widetilde{\mathbb{A}}^{2}$, to be the Zariski closure of $\sigma\left(\mathbb{A}^{2} \backslash\{0\}\right)$ in $\mathbb{A}^{2} \times \mathbb{P}^{1}$. It comes along with the projection $\pi: \widetilde{\mathbb{A}}^{2} \rightarrow \mathbb{A}^{2}$.

For a curve $C \subseteq \mathbb{A}^{2}$ the blowup $C^{\prime}$ is the Zariski closure $\overline{\sigma(C \backslash\{0\})} \subseteq \mathbb{A}^{2} \times \mathbb{P}^{1}$. Through this construction a curve $C$ is lifted to a higher-dimensional space where it has more room to spread out such that it becomes smooth 9

A more general definition: A blowup of a smooth variety $W$ is a variety $W^{\prime}$ together with a projection $\pi: W^{\prime} \rightarrow W$, the associated blow-up map. To each

\footnotetext{
${ }^{9}$ For the construction of the blowup of $C$ we used secants. We could also associate to each point $\left(a_{1}, a_{2}\right)$ on $C$ the tangent line. If we use this transformation, known as the Nash modification of $C$, it is a priori only defined for points on $C$ and not on the whole plane $\mathbb{A}^{2}$. However, one can show that the Nash modification corresponds to blowing up the Jacobian ideal of the curve, i.e., the ideal generated by the partial derivatives of the defining equation, and is therefore defined on the whole plane; see, for example, [32.

Nash modifications are an alternative to blowing up, but they are not easy to handle in higher dimensions; see 32 ,36 for a general treatment and 43 for a generalization of Nash modifications.
} 
blowup a center $Z$, a closed subvariety of $W$, is attached, which determines the blowup completely. The center $Z$ is the locus of points above which $\pi$ is not an isomorphism.

In order to obtain the most explicit description of the map $\pi: W^{\prime} \rightarrow W$ we work locally. We consider the neighborhood of a point $a$ in $Z$, where we may assume that $W=\mathbb{A}^{n}$ and that $a=0$ is the origin. Then $Z=V(I)$ is the zeroset of an ideal $I=\left(g_{1}, \ldots, g_{k}\right) \subseteq K\left[x_{1}, \ldots, x_{n}\right]$. The blowup $\widetilde{\mathbb{A}}^{n}$ of $\mathbb{A}^{n}$ with center $Z$ is defined as the Zariski closure of the graph of the map

$$
\sigma: \mathbb{A}^{n} \backslash Z \rightarrow \mathbb{P}^{k-1}, p \mapsto\left(g_{1}(p): \ldots: g_{k}(p)\right),
$$

which lives in $\widetilde{\mathbb{A}}^{n} \subseteq \mathbb{A}^{n} \times \mathbb{P}^{k-1}$. It is easy to show that different choices of generators of $I$ lead to isomorphic results. We call the projection $\pi: \widetilde{\mathbb{A}}^{n} \rightarrow \mathbb{A}^{n}$ on the first factor the blow-up map of $\mathbb{A}^{n}$ with center $Z$ (or with center $I$ ), and the preimage $\mathbb{E}=\pi^{-1}(Z)=Z \times \mathbb{P}^{k-1}$ the exceptional locus. The morphism $\pi$ contracts the second factor $\mathbb{P}^{k-1}$ to the center along the exceptional locus $\mathbb{E}$.

The blowup $\widetilde{\mathbb{A}}^{n}$ can be described by the projective equations

$$
y_{i} g_{j}=g_{i} y_{j},\left(y_{1}: \ldots: y_{k}\right) \in \mathbb{P}^{k-1}, i, j \leq k .
$$

One can cover $\widetilde{\mathbb{A}}^{n}$ by $k$ affine charts, each corresponding to a generator of $I$. The coordinate ring of the $i$-th chart is given by

$$
K\left[x_{1}, \ldots, x_{n}, \frac{g_{1}}{g_{i}}, \ldots, \frac{g_{k}}{g_{i}}\right] .
$$

The inverse of an affine chart expression of a blowup is called a blowdown or contraction.

Resolution. There are various notions of resolution in use. We start with the simplest form, the nonembedded resolution of singularities. A nonembedded resolution of singularities of an algebraic variety $X$ is a proper birational map $\varphi: \widetilde{X} \rightarrow X$ with $\widetilde{X}$ smooth, such that $\varphi$ is an isomorphism over $X \backslash \operatorname{Sing}(X)$.

Let $X$ be a singular variety embedded in a smooth ambient variety $W$. An embedded resolution of singularities of $X$ is a proper birational morphism $\varphi: W^{\prime} \rightarrow$ $W$ where $W^{\prime}$ is also smooth and satisfies the following condition:

(i) The strict transform $X^{\prime}$ of $X$ is smooth and transversal to the exceptional locus in $W^{\prime}$. This means that the total transform of $X$ in $W^{\prime}$ has only normal crossings.

One can impose additional requirements on the embedded resolution; see, for example, 15. Two of the requirements used in the text are the so-called explicitness and equivariance:

(ii) $\varphi$ is a composition of blowups of $W$ in smooth closed centers $Z$ transversal to the exceptional loci.

(iii) If a group acting on $W$ is also acting on $X \subseteq W$, then the action can be lifted to one on $X^{\prime} \subseteq W^{\prime}$.

Consider a resolution $\pi: \widetilde{X} \rightarrow X$ of a surface $X$ with exceptional divisor $\mathbb{E}=$ $\pi^{-1}(\operatorname{Sing}(X))$. Then $\mathbb{E}=\mathbb{E}_{1} \cup \ldots \cup \mathbb{E}_{s}$, where the $\mathbb{E}_{i}$ are irreducible components. We call $\pi$ a good resolution if $\mathbb{E}$ is a normal crossings divisor, i.e., $\mathbb{E}=\mathbb{E}_{1} \cup \ldots \cup \mathbb{E}_{s}$, where each $\mathbb{E}_{i}$ is smooth, the $\mathbb{E}_{i}$ intersect each other transversally and no three components meet: $\mathbb{E}_{i} \cap \mathbb{E}_{j} \cap \mathbb{E}_{k}=\emptyset$ for different $i, j, k$. A good resolution is not unique. One can always achieve a good resolution by additional blowups. 
A resolution $\pi: \widetilde{X} \rightarrow X$ is called minimal if for any other resolution $\pi^{\prime}: \widetilde{X}_{1} \rightarrow X$ there exists a unique morphism $\rho: \widetilde{X}_{1} \rightarrow M$ such that $\pi^{\prime}=\pi \circ \rho$. A minimal resolution is not necessarily good.

\section{ACKNOWLEDGMENTS}

Thanks to: Ágnes Szilárd and Josef Schicho for very helpful discussions, and Dennis Westra for his many comments and his help to shape the article. We are indebted to the anonymous referee for many valuable suggestions to improve the exposition.

\section{ABOUT THE AUTHORS}

Herwig Hauser studied in Paris with Teissier and Douady. His interests include resolution of singularities, local analytic varieties, infinite dimensional geometry, and vizualization of surfaces. He is a professor at the University of Vienna. Eleonore Faber is currently writing her Ph.D. dissertation about singularity theory and local analytic geometry under the supervision of Herwig Hauser at the University of Vienna.

\section{REFERENCES}

[1] V. I. Arnol'd. Singularity theory. Selected papers, volume 53 of Mathematical Society Lecture Note Series. Cambridge University Press, Cambridge-New York, 1981. MR631683 (83d:58016)

[2] E. Bierstone and P. Milman. Canonical desingularization in characteristic zero by blowing up the maximum strata of a local invariant. Invent. Math., 28(2):207-302, 1997. MR1440306 (98e:14010)

[3] A. M. Bravo, S. Encinas, and O. Villamayor. A simplified proof of desingularization and applications. Rev. Mat. Iberoamericana, 21(2):349-458, 2005. MR.2174912 (2006h:14018)

[4] T. de Jong and G. Pfister. Local Analytic Geometry. Advanced Lectures in Mathematics. Vieweg, Braunschweig - Wiesbaden, 2000. MR.1760953 (2001c:32001)

[5] A. Dimca. Topics on Real and Complex Singularities. Advanced Lectures in Mathematics. Vieweg, Braunschweig - Wiesbaden, 1987. MR1013785 (92d:32048)

[6] A.-H. Durfee. Fifteen characterizations of rational double points. L'Enseignement Mathématique, 25:131-163, 1979. MR.543555 (80m:14003)

[7] S. Encinas and H. Hauser. Strong resolution of singularities in characteristic zero. Comment. Math. Helv., 77(4):821-845, 2002. MR 1949115 (2004c:14021)

[8] R. Ephraim. Isosingular loci and the Cartesian product structure of complex analytic singularities. Trans. Amer. Math. Soc, 241:357-371, 1978. MR.492307 (80i:32027)

[9] E. Faber. Torische Varietäten, Explosionen und das Newtonpolyeder. Master's thesis, Universität Innsbruck, 2007.

[10] W. Fulton and R. MacPherson. A compactification of configuration spaces. Ann. of Math. (2), 139:183-225, 1994. MR 1259368 (95j:14002)

[11] M. Goresky and R. MacPherson. Intersection homology theory. Topology, 19(2):135-162, 1980. MR.572580 (82b:57010)

[12] G.-M. Greuel. Deformation und Klassifikation von Singularitäten und Moduln. Deutsch. Math.-Verein. Jahresber. Jubiläumstagung 1990, pages 177-238, 1992.

[13] R. Hartshorne. Algebraic Geometry, volume 52 of Graduate Texts in Mathematics. Springer, New York, 1977. MR0463157 (57:3116)

[14] H. Hauser. Excellent surfaces and their taut resolution. In Resolution of Singularities, Progress in Math., volume 181. Birkhäuser, 2000. MR1748627 (2001f:14028)

[15] H. Hauser. The Hironaka Theorem on resolution of singularities (Or: A proof that we always wanted to understand). Bull. Amer. Math. Soc., 40:323-403, 2003. MR.1978567(2004d:14009)

[16] H. Hauser and T. Gaffney. Characterizing singularities of varieties and of mappings. Invent. Math., 81(3):427-447, 1985. MR807067 (87m:32019) 
[17] H. Hauser and G. Müller. The trivial locus of an analytic space map germ. Annales de l'Institut Fourier, 39(4):831-844, 1989. MR1036334 (91m:32035)

[18] H. Hironaka. Resolution of singularities of an algebraic variety over a field of characteristic zero, I, II. Ann. of Math. (2), 79:109-326, 1964. MR0199184 (33:7333)

[19] F. Hirzebruch. Über vierdimensionale Riemannsche Flächen mehrdeutiger analytischer Funktionen von zwei komplexen Veränderlichen. Math. Ann., 126:1-22, 1953. MR0062842 (16:26d)

[20] H. Jung. Darstellung der Funktionen eines algebraischen Körpers zweier unabhängiger Veränderlicher $x, y$ in der Umgebung einer Stelle $x=a, y=b$. J. Reine Angew. Math., 133:289-314, 1908 .

[21] H. Jung. Über ganze birationale Transformationen der Ebene. J. Reine Angew. Math., 184:161-174, 1942. MR0008915 (5:74f)

[22] D. Kirby. The structure of an isolated multiple point of a surface II. Proc. London Math. Soc., 7:1-18, 1957. MR0087203(19:319c)

[23] H. Kraft. Geometrische Methoden in der Invariantentheorie. Aspekte der Mathematik. Vieweg, Braunschweig-Wiesbaden, 2nd edition, 1985. MR768181 (86j:14006)

[24] H. Kraft and G. Schwarz. Finite automorphisms of affine $n$-space. In Automorphisms of affine spaces (Curaçao,1994), pages 55-66, Kluwer Acad. Publ., Dordrecht, 1995. MR1352690 (96i:14014)

[25] K. Lamotke. Regular solids and isolated singularities. Advanced Lectures in Mathematics. Vieweg, Braunschweig - Wiesbaden, 1986. MR845275 (88c:32014)

[26] H. Laufer. Normal Two-dimensional Singularities, volume 71 of Annals of Math. Studies. Princeton Univ. Press, 1971. MR0320365 (47:8904)

[27] L. Li. Wonderful compactification of an arrangement of subvarieties. Michigan Math. J. 58/2, 2009.

[28] J. Lipman. Rational singularities, with applications to algebraic surfaces and unique factorization. Publ. Math. IHES, 36:195-279, 1969. MR0276239(43:1986)

[29] J. Lipman. Introduction to resolution of singularities. In Proc. Sym. Pure Math., volume 29, pages 187-230, Arcata, 1974. MR0389901 (52:10730)

[30] J. N. Mather and S. S.-T. Yau. Classification of Isolated Hypersurface Singularities by their Moduli Algebras. Invent. Math., 69:243-251, 1982. MR674404 (84c:32007)

[31] A. Némethi. Five lectures on normal surface singularities. In K. Böröczky, W. Neumann, and A. Stipsic, editors, Low Dimensional Topology, volume 8 of Bolyai Society Math. Studies, pages 269-351. János Bolyai Mathematical Society, 1999. MR1747271 (2001g:32066)

[32] A. Nobile. Some properties of the Nash blowing-up. Pacific J. Math., 60:297-305, 1975. MR0409462 (53:13217)

[33] V. L. Popov. On polynomial automorphisms of affine spaces. Izv. Ross. Akad. Nauk Ser. Mat., 65(3):153-174, 2001. MR 1853370 (2002g:14092)

[34] J. Rosenberg. Blowing up nonreduced subschemes of $\mathbb{A}^{n}$. preprint, 1998.

[35] I. P. Shestakov and U. Umirbaev. The tame and the wild automorphisms of polynomial rings in three variables. J. Amer. Math. Soc., 17(1):197-227, 2004. MR2015334(2004h:13022)

[36] M. Spivakovsky. Sandwiched singularities and desingularization of surfaces by normalized Nash transformations. Ann. of Math. (2), 131:411-491, 1990. MR.1053487 (91e:14013)

[37] B. Teissier. Introduction to equisingularity problems. In Proc. Sym. Pure Math., volume 29, pages 593-632, 1974. MR0422256 (54:10247)

[38] D. Trotman. Lectures on Real Stratification Theory. In A. Pichon, editor, Proceedings Winter School on Resolution of Singularities Marseille, pages 139-155, 2005. MR2342910 (2008g:58009)

[39] W. van der Kulk. On polynomial rings in two variables. Nieuw Arch. Wisk., 1:33-41, 1953. MR0054574 (14:941f)

[40] O. Villamayor. Patching local uniformizations. Ann. Sci. École Norm. Sup.(4), 25(6):629677, 1992. MR:1198092 (93m:14012)

[41] H. Whitney. Local properties of analytic varieties. In Differential and Combinatorial Topology, pages 205-244. Princeton Univ. Press, 1965. MR0188486 (32:5924)

[42] H. Whitney. Tangents to an analytic variety. Ann. of Math. (2), 81(2):496-546, 1965. MR0192520 (33:745)

[43] T. Yasuda. Higher Nash blowups. Compositio Math., 143:1493-1510, 2007. MR2371378 (2008j:14029) 
[44] O. Zariski. The reduction of singularities of an algebraic surface. Ann. of Math (2), 40:639689, 1939. MR0000159(1:26d)

[45] O. Zariski. Contributions to the problem of equisingularity. CIME, Rome, pages 261-343, 1970. MR0276240(43:1987)

Fakultät für Mathematik, Universität Wien, Austria

E-mail address: eleonore.faber@univie.ac.at

Fakultät für Mathematik, Universität Wien, Austria

E-mail address: herwig.hauser@univie.ac.at 